\title{
El deber de gestión del riesgo de corrupción en la empresa emanado de la ley 20393 de Chile: especial referencia a las exigencias de identificación y evaluación de riesgo* The Duty to Manage the Risk of Corruption in the Company Emanated from Chilean Law 20393: Special Reference to the Identification and Risk Assessment Requirements
}

\author{
OSVALDO ARTAZA* \\ Universidad de Talca
}

\section{SEBASTIÁN GALLEGUILLOS** Universidad de Talca}

Resumen: El presente artículo da cuenta de uno de los principales problemas asociados a la atribución de responsabilidad penal a personas jurídicas por la comisión de un delito de cohecho por parte de uno de sus integrantes, cual es el de otorgar criterios para determinar cuándo tal sujeto de imputación ha infringido su deber de gestionar los riesgos de soborno que genera en el marco del desarrollo de su actividad económica. Se ha puesto especial énfasis en la delimitación de tal deber y, específicamente, en la concreción de las exigencias de identificación de tales riesgos con el propósito de adoptar medidas para poder contrarrestarlos con posterioridad. El artículo analiza la experiencia comparada: tanto en lo relativo a las directrices técnicas que provienen de organismos dedicados al área del compliance, como con respecto al aporte de la criminología del delito corporativo. Esto tiene como objeto identificar una serie de variables, tanto estructurales como culturales, de las organizaciones que harían previsible la comisión de tal clase de delitos y, por ende, exigible su identificación por parte de la empresa.

Palabras clave: responsabilidad penal de personas jurídicas, cultura organizacional, gestión de riesgos penales, identificación y evaluación de riesgos, factores de riesgo criminógenos

Abstract: This article analyzes the criteria to determine when a company has failed to meet it is duty to manage the risks of bribery that arise over the course of it is economic activity. Special emphasis has been placed on the

* Trabajo realizado en el marco del proyecto Fondecyt número 1150148 «Corrupción y actividad empresarial. Análisis conceptual para una nueva sistematización». Agradecemos a Mario Orellana Zenteno por su valiosa colaboración en la preparación de este artículo.

** Doctor en Derecho y Ciencias Políticas (Universidad de Barcelona) y Profesor de Derecho Penal (Universidad de Talca).

Código ORCID: 0000-0001-8453-5069. Correo electrónico: oartaza@ utalca.cll

*** Abogado, Licenciado en Ciencias Jurídicas y Sociales (Universidad de Chile). Estudiante del Master of Arts in International Crime \& Justice, John Jay College of Criminal Justice (CUNY). Investigador del Centro de Estudios de Derecho Penal (Universidad de Talca).

Código ORCID: 0000-0001-5772-637X. Correo electrónico: sgalleguillos@utalca.cl 
establishment of requirements for identifying such risks with the purpose of being able to adopt measures to counteract them later. The article analyzes comparative experience, both in relation to the technical guidelines that come from compliance studies, as well as the studies of criminology of corporate crime. Those experiences are explored to identify a series of structural and cultural variables that would predict the commission of crimes and, therefore, require their identification by the company.

Key words: corporate crime liability, organizational culture, management and assessment of risks, criminogenic risk factor

CONTENIDO: I. INTRODUCCIÓN.- II. LÍMITES ASOCIADOS A LA ACTUACIÓN EN BENEFICIO DE LA PERSONA JURÍDICA: ESPECIAL REFERENCIA A LA EXIGENCIA DELVIINCULO CORPORATIVO EN EL ÁMBITO ANGLOAMERICANO.III. EL CUMPLIMIENTO DE LOS DEBERES DE DIRECCIÓN Y SUPERVISIÓN: EXIGIBILIDAD EN LA GESTIÓN DEL RIESGO PENAL POR PARTE DE LA PERSONA JURÍDICA.- III.1. PUNTO DE PARTIDA: PARÁMETROS MÍNIMOS EXIGIDOS POR EL LEGISLADOR PARA LA GESTIÓN DE RIESGOS PENALES.III.2. LA NECESARIA REFERENCIA A LA REGULACIÓNTÉCNICA O DIRECTRICES EN EL ÁREA DE LA GESTIÓN DE RIESGOS DE INCUMPLIMIENTO DE LEY.- IV. FACTORES DE RIESGO CRIMINÓGENOS ASOCIADOS A LA CORRUPCIÓN PROPIOS DE LA ACTIVIDAD EMPRESARIAL.- IV.1. OPORTUNIDADES DELICTIVAS EN CONTEXTOS ORGANIZACIONALES.- IV.2. TÉCNICAS DE NEUTRALIZACIÓN Y CULTURA EMPRESARIAL.- V. CONCLUSIONES.

\section{INTRODUCCIÓN}

Una de las principales tendencias en la actualidad, en lo que se refiere a la prevención o lucha contra la corrupción, es la incorporación del sector privado, específicamente el empresarial, como un sector estratégico para tal labor (García, 2016, p. 220; Nieto, 2013, p. 192) ${ }^{1}$. Esta realidad ha generado una serie de cambios culturales en lo que respecta a la gestión de organizaciones, pero además, y como se podrá apreciar, ha implicado importantes adecuaciones a los ordenamientos jurídicos, ya que tal incorporación se ha efectuado, por lo general, a través del surgimiento de nuevos deberes dirigidos a las personas jurídicas. Estos nuevos deberes suponen adoptar los mecanismos necesarios para evitar que, a propósito de su actividad económica, se efectúen actos

1 Al hablar de corrupción o actos de corrupción, nos referimos exclusivamente al fenómeno del "soborno» a funcionarios públicos nacionales o extranjeros, cualquiera que sea la modalidad que este adopte y que se encuentra recogido en la legislación chilena, a través de la tipificación del delito de cohecho en los artículos 249 al 251 bis del Código Penal chileno. En el ámbito de la adopción de sistemas antisoborno, destaca la definición otorgada por la norma ISO 37001, según la cual debe entenderse por soborno la «oferta, promesa, entrega, aceptación o solicitud de una ventaja indebida de cualquier valor (que puede ser de naturaleza financiera o no financiera), directamente o indirectamente e independiente de su ubicación en violación de la ley aplicable, como incentivo o recompensa para que una persona actúe o deje de actuar en relación con el desempeño de las obligaciones de esa persona» (ISO 37001:2016, punto 3.1). 
de corrupción, especialmente en el marco de sus interacciones con funcionarios del Estado (para un panorama general de la cuestión en América Latina, véase Organización para la Cooperación y el Desarrollo Económicos (OECD), 2013b). Lo anterior se ha verificado, incluso, a través de la adopción, por distintos ordenamientos jurídicos, de sistemas de atribución de responsabilidad penal a las personas jurídicas por los delitos de cohecho cometidos por sus integrantes en el desempeño de sus funciones.

Es así como en Chile, a partir de la entrada en vigencia de la ley 20393, las personas jurídicas pueden responder penalmente por el delito de cohecho, en caso de que se cumplan ciertos requisitos contenidos en la misma regulación. Las exigencias básicas para la atribución de responsabilidad penal a las personas jurídicas se encuentran contenidas en el artículo 3 de este cuerpo legal, que dispone lo siguiente:

Artículo 3ํ.- Atribución de responsabilidad penal.

Las personas jurídicas serán responsables de los delitos señalados en el artículo $1^{\mathrm{o}}$ que fueren cometidos directa e inmediatamente en su interés o para su provecho, por sus dueños, controladores, responsables, ejecutivos principales, representantes o quienes realicen actividades de administración y supervisión, siempre que la comisión del delito fuere consecuencia del incumplimiento, por parte de ésta, de los deberes de dirección y supervisión.

Bajo los mismos presupuestos del inciso anterior, serán también responsables las personas jurídicas por los delitos cometidos por personas naturales que estén bajo la dirección o supervisión directa de alguno de los sujetos mencionados en el inciso anterior.

Se considerará que los deberes de dirección y supervisión se han cumplido cuando, con anterioridad a la comisión del delito, la persona jurídica hubiere adoptado e implementado modelos de organización, administración y supervisión para prevenir delitos conforme a lo dispuesto en el artículo siguiente.

Las personas jurídicas no serán responsables en los casos que las personas naturales indicadas en los incisos anteriores, hubieren cometido el delito exclusivamente en ventaja propia o a favor de un tercero.

Como se desprende de la mera lectura de este enunciado, la persona jurídica no responde automáticamente por la mera comisión de un delito de cohecho por parte de uno de sus integrantes ${ }^{2}$. Por lo mismo, parte de

2 Este punto es del todo relevante, ya que se relaciona con un aspecto de suma importancia denominado por la doctrina especializada el «modelo de imputación», de responsabilidad penal a las personas jurídicas. Este es un tema que no puede ser abordado acá, debido a que excede con creces los objetivos de este trabajo. Basta señalar que la doctrina chilena estaría de acuerdo en que

EL DEBER DE GESTIÓN DEL RIESGO DE CORRUPCIÓN EN LA EMPRESA EMANADO

DE LA LEY 20393

DE CHILE: ESPECIAL

REFERENCIA A LAS

EXIGENCIAS DE

IDENTIFICACIÓN

Y EVALUACIÓN DE

RIESGO

THE DUTYTO

MANAGETHE RISK

OF CORRUPTION

IN THE COMPANY

EMANATED FROM

CHILEAN LAW

20393: SPECIAL

REFERENCETOTHE

IDENTIFICATION

AND RISK

ASSESSMENT REQUIREMENTS 
la doctrina chilena ha señalado que nuestro sistema de responsabilidad penal corporativa satisface ciertos límites a la utilización de la sanción penal, como es el de la prohibición de la responsabilidad objetiva o la responsabilidad por el hecho ajeno (Hernández, 2010, p. 213). Sin embargo, uno de los principales desafíos interpretativos que se le plantea a la dogmática penal se refiere a la concreción del «injusto» por el cual responde la empresa, ya que, como resulta obvio, el delito de cohecho en particular es cometido por uno de sus integrantes, el que también puede ser sancionado penalmente al mismo tiempo que a la persona jurídica. De lo anterior se desprende que resulta necesario determinar cuál es el vínculo entre persona jurídica y delito que justifica, de acuerdo con la normativa vigente, la sanción penal a la empresa en particular.

El primer vínculo exigido por la ley se colige del hecho de que la organización solo pueda ser sancionada en los casos en que el delito sea cometido por algunos de sus integrantes, siempre que lo hayan cometido directa e inmediatamente en su interés. Tal requisito puede ser denominado como el del "vínculo corporativo»" del cual se extrae que la empresa podría responder exclusivamente de verdaderos casos de «corrupción en los negocios», entendiéndose por tal todo acto de cohecho o soborno en el que interviene un integrante de la empresa que recurre a dicha conducta como una forma de propender a la consecución de los objetivos corporativos. El legislador chileno ha querido dejar en claro que la empresa no debe responder frente a casos en que el delito deba ser entendido como un hecho externo a su actividad, lo que resulta evidente, al menos, cuando el delito se comete exclusivamente en beneficio del autor o de un tercero ${ }^{4}$.

Para entender esta clase de vínculo, se debe tener en consideración que cualquier actividad económica, efectuada a través de una persona jurídica, conllevaría, al menos y en forma muy genérica, dos clases de riesgos. El primero de estos es que la entidad sea instrumentalizada por un tercero o un integrante para cometer un delito en su propio provecho, del que incluso la empresa puede ser víctima. Un segundo grupo de riesgos está dado por los casos en que los integrantes de la misma organización

no se trataría de un sistema de responsabilidad vicarial o por transferencia puro, esto es, un sistema en que la empresa responde exclusivamente por la actuación de sus integrantes y en donde se traspasaría sin más la responsabilidad de estos a la primera (Artaza, 2013a, pp. 83ss.), sino que, más bien, respondería por su propio defecto de organización. Sin embargo, existe cierto nivel de disenso en el medio chileno ya que, para algunos, nuestro sistema correspondería a un «modelo atenuado de responsabilidad derivada» (Hernández, 2010, p. 216), mientras que, para otros, a un sistema de autorresponsabilidad o verdadera responsabilidad por el hecho propio (Balmaceda \& Guerra, 2014, p. 98) o, incluso, a un sistema que funda la responsabilidad en el carácter (Mañalich, 2011, pp. 296ss.).

3 Como se señalará más adelante, cierta práctica comparada exige, en forma más precisa, un vínculo comercial, así, por ejemplo, en la legislación estadounidense y la del Reino Unido.

4 El problema de la actuación en interés de la empresa es un problema básico para la atribución de responsabilidad penal corporativa, siendo incluso el principal requisito de los sistemas de responsabilidad vicarial o por transferencia (Artaza, 2013a, pp. 94ss.). 
traten de obtener o alcanzar ciertos objetivos corporativos aun a costa del incumplimiento de la ley, por ejemplo, a través de la comisión de un delito. Como se podrá observar a continuación, es un elemento común a esta tendencia internacional de sancionar a la empresa por actos de corrupción el restringir la aplicación de la responsabilidad penal a este segundo grupo de casos. Con el objeto de ahondar en este vínculo, se analizará cierta experiencia en el ámbito comparado relativa a la determinación de cuándo un pago efectuado por un integrante de la empresa puede entenderse como un acto de corrupción en los negocios. El desarrollo más patente de este vínculo se debe, sin duda alguna, al ámbito angloamericano, tanto a través del desarrollo de la ley del Congreso de los Estados Unidos, Foreign Corrupt Practices Act (en adelante FCPA), como de la ley de 2010 del Reino Unido, Bribery Act (en adelante BA 2010). En ambos casos, aunque se presenten ciertas diferencias entre tales instrumentos, se reserva la sanción penal a los supuestos en que se satisfaga el requisito de la presencia de un vínculo comercial entre delito y la empresa.

Pero, además, para que la empresa pueda ser sancionada por la comisión de tales delitos, se exige un vínculo adicional, que justamente es el que permitiría sostener que en ningún caso el sistema chileno de atribución de responsabilidad penal corporativa pueda ser interpretado como un sistema de mera transferencia de responsabilidad de una conducta de un integrante, aunque se haya efectuado en beneficio de la misma. Como señala el artículo 3 de la ley 20393, la empresa solo responde en los casos en que el delito sea una consecuencia del incumplimiento, por parte de la persona jurídica, de sus deberes de dirección y supervisión ${ }^{5}$. Lo anterior es de suma relevancia, ya que de esto se colige que, en determinados supuestos, la empresa no respondería penalmente, esto es: si se trata de delitos que no se hubieran podido prevenir aun habiendo cumplido tales deberes ${ }^{6}$.

5 Es muy importante evitar el malentendido de creer que la empresa responde penalmente simplemente por no haber adoptado e implementado un sistema de organización, administración y supervisión para prevenir un delito de cohecho, ya que nuestro sistema exige constatar que tal omisión es la que permite o explica la comisión del delito. Por ende, este artículo pretende ser un aporte a la resolución del problema del vínculo que presenta la omisión de tales deberes y la previsibilidad de comisión de delitos en el seno de la empresa. Para lo cual se analizará especialmente los aportes que provienen de diversos organismos técnicos dedicados a la gestión del riesgo, especialmente el de incumplimiento de la ley, y de la literatura criminológica especializada en el delito corporativo y en explicar sus causas $u$ orígenes.

6 Incluso parte de nuestra doctrina ha señalado que la empresa no podría responder en caso del cumplimiento de tales deberes, ya que lo anterior demostraría que «la persona jurídica no ha generado más riesgos que los que el derecho le permite generar» (Piña, 2012, p. 7). Por eso, en el ámbito comparado, ha adquirido suma relevancia la posibilidad de defensa de la empresa por haber adoptado sistemas de prevención de tales delitos (conocida, en el ámbito angloamericano, como "compliance defense»). En sistemas como el estadounidense, donde no se encuentra especialmente reconocida tal defensa, se ha abogado por su inclusión, sin perjuicio de que se haya señalado que existiría cierto nivel de reconocimiento tácito de la misma (Koehler, 2012, pp. 631ss.; Jordan, 2012, pp. 103ss.). 
Tal disposición resulta fundamental para delimitar los criterios atributivos de responsabilidad penal a las personas jurídicas en Chile, en la medida en que de aquí se extraería un verdadero mandato de cumplimiento de un deber de dirección y supervisión, el que, además, no resulta fácil de concretar. Como se podrá apreciar en este artículo, al menos en cierta práctica comparada, este deber de dirección y supervisión puede ser entendido como un mandato de gestión de riesgos de incumplimiento de la ley penal por parte de los integrantes de la organización. Con todo, uno de los principales problemas que se plantearán y que pretenden ser desarrollados acá, dice relación con la delimitación de la clase de riesgos que la empresa debe gestionar. Para tales efectos se recurrirá, en primer lugar, al análisis de los criterios otorgados por la propia ley 20393, especialmente en su artículo 4 que define cuándo este deber se entiende cumplido.

A partir de lo enunciado por esta disposición, se desprende que la empresa solo debe gestionar aquellos riesgos de comisión del delito de cohecho que sean previsibles en el marco del desarrollo de su actividad. Como se podrá apreciar, tal requisito de exigibilidad resulta sumamente complejo de delimitar en este ámbito, ya que para la gestión del riesgo de comisión de un delito de cohecho, no solo se deben tener en cuenta algunas directrices relativas a la gestión del riesgo como problema general del gobierno corporativo, sino que también y, sobre todo, la concurrencia de ciertos factores de riesgo que pueden presentarse en el desarrollo de toda actividad empresarial y que pueden incidir en distintas formas en que se cometa un delito de cohecho para la consecución de los objetivos corporativos o que estos no sean detectados en forma oportuna. En definitiva, para gestionar tales riesgos, se debe considerar una serie de factores tanto externos como internos a la propia compañía que pueden fomentar la comisión de tales delitos, ya sea en forma separada o en conjunción entre unos y otros. Pero, por otra parte, se debe tener también en cuenta factores de riesgo asociados a la propia organización y su estructura o control interno que pueden impedir o dificultar la detección y respuesta oportuna frente a la comisión de un delito de cohecho. Por lo mismo, se entiende que el núcleo del deber de gestión de riesgos contemple no solo la identificación y evaluación de riesgos específicos para la entidad y la actividad que desarrolla, sino también la adopción de medidas de control o de respuesta a tales riesgos y el diseño de un sistema de supervisión de la ejecución de tales medidas y su modificación en caso de ser necesario.

En suma, el propósito de este artículo es contribuir a resolver uno de los aspectos que resultan fundamentales a la hora de comprender el deber de supervisión y dirección que emana de la legislación chilena y que recaería en la persona jurídica, proporcionando criterios rectores para delimitar qué clase de riesgos deben ser contrarrestados por la 
organización, ya que resulta previsible que la omisión de tal gestión pueda incidir o facilitar la comisión de un delito de cohecho en el marco del desarrollo de su actividad económica. En la medida en que se reconozca tal previsibilidad resulta, entonces, exigible a la organización adoptar medidas para reducir las posibilidades de concreción de tal ilícito?

\section{LÍMITES ASOCIADOS A LA ACTUACIÓN EN BENEFICIO DE LA PERSONA JURÍDICA: ESPECIAL REFERENCIA A LA EXIGENCIA DEL VÍNCULO CORPORATIVO EN EL ÁMBITO ANGLOAMERICANO}

Como se ha señalado previamente, la empresa solo responde penalmente en caso de que infrinja el mandato que surge de la ley 20393 de gestionar determinados riesgos que derivan del desarrollo de su propia actividad. Un principio general y básico de la gestión de riesgos es el de la identificación previa de los mismos, con el objeto de evaluarlos y determinar las medidas o respuestas que se implementarán para contrarrestarlos (Artaza, 2013a, pp. 157ss.). Como resulta evidente, la identificación de riesgos supone conocer en profundidad qué es aquello que se pretende gestionar, lo que en este ámbito no necesariamente resulta sencillo ${ }^{8}$. Uno de los principales límites que se extraen de nuestra regulación es que la empresa solo responde de aquellos casos de corrupción que se cometan directa e inmediatamente en su interés y no, por el contrario, cuando el delito deba ser considerado como un hecho externo, no vinculado a los asuntos corporativos?.

Como señala Hernández, con este requisito se pretende, en primer lugar, evitar que la empresa responda por hechos que «no sean susceptibles de reportarle ningún beneficio», sobre todo cuando en estos actos se pueda incluso terminar perjudicando los intereses económicos de la empresa, excluyendo así aquellos actos que se han cometido en intereses o provecho exclusivo del autor del delito o de un tercero (Hernández, 2010, pp. 220-221). Así las cosas, correspondería determinar qué clase de nexo se podría presentar entre los actos de corrupción y la actuación

7 Por tanto, no se dará cuenta acá, al menos en forma detallada — debido a que excede con creces los objetivos de este trabajo-, del problema relativo a los mecanismos que deben ser adoptados por la persona jurídica para contrarrestar tales riesgos. Solo se analizará la cuestión de la respuesta frente al riesgo en la medida que resulte relevante como problema asociado a la previsibilidad de comisión de delitos en el marco del desarrollo de una actividad económica por una organización.

8 Por lo mismo, organismos internacionales, como, por ejemplo, Transparency International UK, se han dedicado a impartir directrices o criterios para entender qué debe ser entendido por soborno en este ámbito (véase Muravska, 2014).

9 Es importante señalar que este no es el único límite a la gestión de riesgos vinculado al conocimiento del fenómeno que se pretende prevenir —o riesgo que se quiere contrarrestar-, ya que un primer elemento, que no puede ser abordado acá y que ha sido analizado en otro lugar, dice relación con las dificultades asociadas a determinar cuándo se está en presencia, en definitiva, de un caso de un delito de cohecho en el ámbito de los negocios. Así, por ejemplo, se ha discutido, especialmente en la práctica estadounidense, algunos problemas que surgen de ciertos pagos "para facilitar trámites» y pagos efectuados a la contraparte para promocionar un producto o de aquellos conocidos como «gastos de hospitalidad» (Carnevali \& Artaza, 2016, pp. 75ss.).

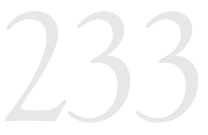

EL DEBER DE GESTIÓN DEL RIESGO DE CORRUPCIÓN EN LA EMPRESA EMANADO DE LA LEY 20393 DE CHILE: ESPECIAL REFERENCIA A LAS EXIGENCIAS DE IDENTIFICACIÓN YEVALUACIÓN DE RIESGO

THE DUTYTO MANAGETHE RISK OF CORRUPTION IN THE COMPANY EMANATED FROM CHILEAN LAW 20393: SPECIAL REFERENCETOTHE IDENTIFICATION

AND RISK

ASSESSMENT REQUIREMENTS 
en interés directo e inmediato de la persona jurídica. Este aspecto es de suma relevancia, en la medida que, como se podrá apreciar a continuación a través de una breve referencia al ámbito comparado, las exigencias de prevención de la corrupción dirigidas a las personas jurídicas tienen por principal objeto evitar que los integrantes de una empresa pretendan alcanzar o facilitar la consecución de los objetivos corporativos a través del pago de sobornos al sector público.

Tal exigencia sería coincidente con la tendencia angloamericana de restringir la sanción a la empresa en casos en que se constate determinado vínculo entre el delito y la actividad económica en particular. Así, tanto en la BA 2010 como en la FCPA se contempla en forma expresa el requisito del «nexo comercial» (business nexus requirement), sin perjuicio de que su alcance no sea exactamente el mismo.

Como se desprende de la observación de tales instrumentos, el vínculo comercial entre la organización y el delito de cohecho puede ser de tres tipos. El más evidente y, por ende, menos problemático, está dado por los casos en que el soborno está dirigido a la obtención o manutención de un negocio. En segundo lugar, se pueden identificar los supuestos en que tal acto de corrupción tenga como propósito colaborar en cualquier forma con la obtención o manutención de un negocio. Por último, se encuentran aquellas situaciones caracterizadas por el hecho de que el beneficio indebido otorgado se efectúe para obtener cualquier clase de ventaja corporativa, facilitando así la consecución ilícita de los objetivos corporativos.

Un buen ejemplo de un sistema en donde se exige un vínculo comercial sumamente fuerte entre el soborno y la empresa es el que se extrae de la FCPA. Como es sabido, y simplificando en extremo este aspecto, a través de este instrumento se sanciona a quienes sobornen a «un funcionario público extranjero, a cualquier partido político extranjero o funcionario de ese partido o a cualquier candidato a un cargo político extranjero» (Carnevali \& Artaza, 2016, p. 69), entre otros, si tal pago se efectúa con el propósito de «influir en cualquier acto o decisión de dicho funcionario; inducirlos a que realicen u omitan algún acto en infracción de su deber legítimo, obtener alguna ventaja indebida, o inducir a dichos sujetos a que ejerzan conductas de tráfico de influencias en los respectivos gobiernos extranjeros para afectar un acto o decisión de dicho gobierno» (Carnevali \& Artaza, 2016, p. 70). Con todo, solo son punibles tales conductas si la prestación correlativa del funcionario está encaminada a ayudar al sobornante a obtener o retener algún negocio para sí mismo o un tercero, o para encaminar algún negocio hacia alguna persona. Tal exigencia fue históricamente vinculada con la demostración de que el soborno fue efectuado en conexión con una 
oferta por un nuevo contrato o la renovación de un contrato existente (Lu, 2013, p. 741).

En la actualidad, se discute el alcance de tal elemento, lo que habría sido potenciado por la ambigüedad del mismo (Koehler, 2010, p. 393), ya que hay quienes sostienen que el soborno debe ser exclusivamente instrumental o efectuado con el fin de obtener la aprobación de un nuevo contrato con el Estado o la renovación de un contrato previamente celebrado. Como explica Lu, bajo tal interpretación no podría considerarse que los casos de soborno para mejorar la rentabilidad de la empresa en la ejecución de un contrato vigente con el Estado cumplan con el requisito del nexo comercial ya descrito (Lu, 2013, p. 741).

Fundamental para entender la evolución de este requisito es tener en cuenta la resolución del caso United States v. Kay. Como explica Lu, se sometió a discusión, a propósito de este caso, si podía entenderse como un pago para obtener o retener negocio el soborno a funcionarios públicos de aduanas de Haití con el propósito de adulterar la información relevante para el pago de impuestos de internación de mercaderías y, así, reducir significativamente tal carga (Lu, 2013, p. 742). En definitiva, se resolvió que el requisito del nexo comercial incluiría pagos distintos a los destinados a obtener o mantener un contrato, ya que también consideraría aquellos que pudieran ayudar de cualquier forma, directa o indirectamente a obtener o mantener un negocio ${ }^{10}$. Así, por ejemplo, y como razona el tribunal respectivo, tales pagos para reducir impuestos podrían permitir que la empresa sobornante efectúe una mejor oferta que sus competidores, disminuya las posibilidades de quiebra de la misma, o mejore las posibilidades para competir y obtener nuevos contratos (Lu, 2013, p. 743). Incluso como explica cierta doctrina, hay quienes sostienen que este caso habría ampliado el campo de este requisito a cualquier pago destinado a conseguir una ventaja en comparación al resto de los competidores o mejorar las oportunidades comerciales (Pacini, 2012, p. 568; Dunst, Diamant \& Kung, 2011, pp. 269-270; Lu, 2013, p. 743).

El problema interpretativo recientemente planteado no se presenta en el caso de la normativa del Reino Unido, debido a que en la sección 7 de la BA 2010 se sanciona la infracción del deber de prevenir la corrupción para determinadas organizaciones comerciales reguladas por la ley del Reino Unido y que realizan, al menos, parte de sus actividades dentro de su territorio (Bean \& MacGuidwin, 2013, p. 86), si uno de sus asociados

10 En estricto rigor, como explica Koehler, el tribunal sostuvo que el Congreso de Estados Unidos, en su momento, pretendió que este instrumento se aplicara en general a todo pago que se realizara con el propósito de ayudar al sobornante directa o indirectamente a obtener o retener un negocio y que, en algunas ocasiones, los pagos efectuados a funcionarios extranjeros para reducir cargas o gravámenes tributarios podrían caer perfectamente en tal categoría (Koehler, 2010, p. 393).

EL DEBER DE GESTIÓN DEL RIESGO DE CORRUPCIÓN EN LA EMPRESA EMANADO DE LA LEY 20393 DE CHILE: ESPECIAL REFERENCIA A LAS EXIGENCIAS DE IDENTIFICACIÓN YEVALUACIÓN DE RIESGO

THE DUTYTO MANAGETHE RISK OF CORRUPTION IN THE COMPANY EMANATED FROM CHILEAN LAW 20393: SPECIAL REFERENCETOTHE IDENTIFICATION AND RISK ASSESSMENT REQUIREMENTS 
— considerado en un sentido amplio ${ }^{11}$ — soborna a otra persona con la intención de (a) obtener o retener negocios para la empresa o, (b) obtener o retener una ventaja en el desarrollo de un negocio para tal empresa (Alldridge, 2012, p. 1201; Rose, 2012, p. 495).

Como se puede observar, para el caso de la comisión de un delito de cohecho, el vínculo o nexo con los intereses de la empresa puede ser variado: obtener o retener negocios con el Estado, facilitar de cualquier forma tal obtención o mantenimiento o, por último, obtener cualquier ventaja indebida a través del pago de un soborno.

De acuerdo con lo señalado con anterioridad, parece claro que el sistema chileno contempla el requisito del nexo comercial en una forma similar a la establecida en la BA 2010. Así, a diferencia de lo que sucede en el ámbito estadounidense, no se puede poner en duda que este nexo comercial puede ser sumamente amplio, es decir, ser satisfecho con la sola constatación de que se soborna a un funcionario público de cualquier forma que resulte idónea para la obtención o manutención de una ventaja para la realización del negocio. Así las cosas, cualquier acto de corrupción que presente tal condición de idoneidad debe ser entendido como cometido en interés directo e inmediato de la persona jurídica. Esto quiere decir que este requisito se debe entender relacionado en forma directa con la posibilidad de que el delito de cohecho esté, en cualquier forma, vinculado al desarrollo del negocio en particular y no deba ser comprendido como un hecho exclusivamente externo sin conexión alguna con los asuntos corporativos.

Así, por ejemplo, la empresa tendría que considerar, dentro de la identificación de sus riesgos, casos tan variados como obtener un permiso de funcionamiento o adjudicarse un contrato con el Estado - hipótesis relacionadas con la obtención o mantenimiento de un negocio- hasta situaciones vinculadas con la mera obtención de ventajas, como podría ser la reducción de cargas, aumento de ayudas o subsidios, reducción o eliminación de multas, así como, incluso, obtener resoluciones judiciales o administrativas a favor de los intereses corporativos. En todos estos casos, la empresa, a través de actos de corrupción, adquiere ventajas indebidas para el desarrollo de su actividad, es decir, está facilitando la consecución de sus objetivos a través del pago de sobornos.

Sin embargo, tal identificación de riesgos no es suficiente. De acuerdo con la redacción de la letra a del numeral 3 del artículo 4 de la regulación

\footnotetext{
11 Según la sección 8 de la BA 2010, una persona asociada es cualquiera que efectúe algún servicio a favor o en nombre de la organización de la que se trate, sin importar la capacidad o calidad con la que los efectúe, por lo mismo, puede tratarse de un empleado, agente o auxiliar (Mukwiri, 2015, p. 24). De hecho, una de las críticas que ha efectuado la doctrina — por ejemplo, Bean y MacGuidwin — es que este aspecto de la regulación de la BA 2010 resulta sumamente ambiguo y expansivo, ya que no queda claro el alcance que podría tener el término «asociado» a la organización (2012, p. 345).
} 
respectiva, una vez que la empresa ha determinado cuáles son los procesos o actividades, habituales o esporádicos, en que se presente el riesgo de comisión de un delito de cohecho con el nexo comercial previamente analizado, le corresponde adoptar aquellas medidas que sean exigibles para supervisar que, en la ejecución de las mismas, los sujetos responsables de tales procesos no incurran en una conducta delictiva. Como se señaló anteriormente, uno de los principales criterios atributivos que se consagran en nuestro régimen de atribución de responsabilidad penal a personas jurídicas es que estas solo responden en caso de que el delito sea consecuencia del incumplimiento de sus deberes de dirección y supervisión.

Asimismo, se señaló que este deber debía ser entendido como un deber de gestión de riesgos de comisión de conductas delictivas, por ende, tal deber de gestión debe contemplar medidas que contrarresten todos aquellos factores que previsiblemente podrían incidir en que, en el contexto en el que se desarrollan los procesos o actividades previamente detectados, se termine cometiendo un delito o no pueda detectarse a tiempo para reaccionar adecuadamente. Como consecuencia, para el caso de que la empresa efectivamente haya cumplido con sus deberes de dirección y supervisión y se cometiera igualmente un delito de cohecho por uno de sus integrantes, no podría atribuírsele responsabilidad penal, entendiéndose que el delito no podría haber sido evitado cumpliendo con el mandato que deriva del artículo 3 de la ley 20393.

Este es uno de los aspectos que presenta mayor complejidad a la hora de delimitar los criterios atributivos de responsabilidad penal a la empresa en Chile, debido a que aún no ha sido completamente abordado, en el medio chileno, el problema de la exigibilidad en la gestión de riesgos penales. Como se analizará a continuación, lo anteriormente planteado debe entenderse en estrecha relación con el problema de la previsibilidad de la comisión de delitos en el marco del desarrollo de una actividad económica en organizaciones y de la presencia de defectos en la gestión de la misma que podrían incidir en su comisión o en dificultar su detección o descubrimiento oportuno.

\section{EL CUMPLIMIENTO DE LOS DEBERES DE DIRECCIÓN Y SUPERVISIÓN: EXIGIBILIDAD EN LA GESTIÓN DEL RIESGO PENAL POR PARTE DE LA PERSONA JURÍDICA}

De acuerdo con lo sostenido previamente, del artículo 3 de la ley 20393 se desprende que la empresa solo responde en los casos que el delito se hubiera podido prevenir a través del cumplimiento de su deber de dirección y supervisión, esto es, a través de la adopción, como lo señala el inciso 3 de la misma disposición, de modelos de organización,

EL DEBER DE GESTIÓN DEL RIESGO DE CORRUPCIÓN EN LA EMPRESA EMANADO DE LA LEY 20393 DE CHILE: ESPECIAL REFERENCIA A LAS EXIGENCIAS DE IDENTIFICACIÓN YEVALUACIÓN DE RIESGO

THE DUTYTO MANAGETHE RISK OF CORRUPTION IN THE COMPANY EMANATED FROM CHILEAN LAW 20393: SPECIAL REFERENCETOTHE IDENTIFICATION AND RISK ASSESSMENT REQUIREMENTS 
administración y supervisión para prevenir delitos como el cometido. Por lo mismo, resulta fundamental delimitar el deber de gestión de riesgos que recae en la empresa, con el propósito de otorgar contenido a este criterio atributivo de responsabilidad penal a través de la determinación de cuándo puede comprenderse un delito corporativo como una consecuencia de tal infracción de deberes.

\section{III.1. Punto de partida: parámetros mínimos exigidos por el legislador para la gestión de riesgos penales}

El contenido nuclear del deber de dirección y supervisión está comprendido en el artículo 4 de la ley 20393, a través del establecimiento de los elementos mínimos que debiera presentar un «sistema de prevención de delitos» para satisfacer las exigencias de gestión de tales riesgos $^{12}$. De tal disposición se extraen importantes consecuencias relativas al alcance o delimitación del deber de gestión de riesgos. La primera de estas, de acuerdo con lo establecido por la letra a del numeral 3 del artículo 4, es que el sistema deberá identificar las actividades o procesos de la entidad, sean habituales o esporádicos, en cuyo contexto se genere o incremente el riesgo de comisión de ciertos delitos.

En segundo lugar, se señala que el sistema debe contar con una serie de medidas para contrarrestar los riesgos que se puedan producir a propósito del desarrollo de tales actividades, así, por ejemplo, establecer «...protocolos, reglas y procedimientos específicos» que permitan que los responsables de ejecutarlas puedan llevarlas a cabo evitando la comisión de un delito. En relación con lo anterior, se exige, además, que se identifiquen los procedimientos de administración y auditoría de los recursos financieros que permitan evitar que tales sujetos los utilicen en el marco de una conducta ilícita.

Con todo, y como se desprende de la normativa en comento, no basta con la identificación de riesgos y la adopción de medidas para prevenir la comisión de delitos, si no se contempla, como tercer elemento, una serie de mecanismos de control que permitan favorecer el cumplimiento de las medidas previamente adoptadas. Por lo mismo, se establece la necesidad de incluir en el sistema de prevención de delitos sanciones para casos de incumplimiento, así como herramientas adecuadas para obtener información relevante relativa al seguimiento de tales obligaciones, dentro de las cuales destaca el establecimiento de un procedimiento de denuncia. En forma adicional, se prescribe la necesidad de establecer

12 Nos referiremos exclusivamente a los componentes de un sistema de prevención, entendiendo que los elementos anteriormente exigidos por el artículo 4 -es decir, el nombramiento de encargado de prevención de delitos y los medios- se comprenden en conexión con este sistema y su ejecución. En todo caso, el objeto de este artículo no es ahondar en detalle en la implementación de tales sistemas, sino analizar un aspecto central de dichos sistemas, a saber, la clase de riesgos que, por su previsibilidad, deben ser considerados por la organización para su gestión. 
métodos efectivos de supervisión de la ejecución de las medidas adoptadas, con el objeto de determinar si deben ser modificadas en caso de resultar insuficientes o actualizadas en caso de presentarse algún cambio relevante de circunstancias ${ }^{13}$.

Como se puede observar, uno de los principales problemas asociados a la delimitación del deber de gestión de riesgos penales es que el legislador solo ofrece parámetros mínimos que deben ser alcanzados, sin indicar la forma en la que tales requerimientos deben ser cumplidos. Así, por ejemplo, establece que el deber de dirección y supervisión implica la identificación de los riesgos, sin señalar los mecanismos que debe adoptar la empresa para tales efectos. Lo anterior no ha sido, en ningún caso, considerado como una desventaja, ya que se ha asumido que quienes desarrollan una actividad en particular son quienes poseen, o debieran poseer, el conocimiento necesario para efectuar tal labor (Coca, 2013, pp. 45-46) ${ }^{14}$. Este aspecto resulta de suma relevancia para el objeto de estudio, ya que, en definitiva, a la hora de determinar si la empresa cumplió o no con su deber de gestión de riesgos, se tendrán que valorar las decisiones adoptadas por la misma para satisfacer los requerimientos básicos exigidos por el legislador. Por lo mismo, resultará fundamental analizar las herramientas de las que se dispone en la actualidad y que dotan de contenido el deber de gestión abordado.

\section{III.2. La necesaria referencia a la regulación técnica o directrices en el área de la gestión de riesgos de incumplimiento de ley}

La tendencia recién descrita no es exclusiva del sistema chileno, sino que se presenta también en el ámbito comparado. Así, por ejemplo, en el Reino Unido la sección 7 de la BA 2010 no solo establece la posibilidad de sancionar a la empresa si un colaborador o asociado soborna a otro con el propósito de obtener o retener un negocio o una ventaja, sino que reconoce que la empresa no siempre debiera ser sancionada y la dota de una «defensa» para los casos en que se compruebe que la organización, pese al hecho particular de soborno del que se trate, contaba con procedimientos establecidos para prevenir que sus asociados cometieran tales conductas (Hunter, 2011, pp. 102-103; Dunst et al., 2011, p. 282;

13 La empresa incluso puede certificar el modelo (artículo 4, numeral 4, letra b) con el propósito de que un externo (inscrito en un registro de la Superintendencia de Valores y Seguros) constate tanto que el contenido del Modelo a certificar satisface los requisitos de nuestra legislación como la efectiva implementación y ejecución del mismo.

14 Por eso ha sido común que la doctrina especializada relacione tales exigencias para la prevención de la corrupción por parte de la empresa con el problema de las nuevas formas como el Estado regula la actividad empresarial aprovechando la capacidad de la misma de autorregularse (Nieto, 2008, p. 4). Por lo general, se señala que estos «programas de cumplimiento», «sistemas de prevención de delitos» o «modelos de organización y gestión» son una manifestación de tal estrategia de regulación donde, al menos, se presenta una mezcla entre componentes de la heterorregulación y la autorregulación (Manacorda, 2014, p. 17; Gómez-Jara, 2012, p. 111).

EL DEBER DE GESTIÓN DEL RIESGO DE CORRUPCIÓN EN LA EMPRESA EMANADO DE LA LEY 20393 DE CHILE: ESPECIAL REFERENCIA A LAS EXIGENCIAS DE IDENTIFICACIÓN YEVALUACIÓN DE RIESGO

THE DUTYTO MANAGETHE RISK OF CORRUPTION IN THE COMPANY EMANATED FROM CHILEAN LAW 20393: SPECIAL REFERENCE TO THE IDENTIFICATION

AND RISK ASSESSMENT REQUIREMENTS 
Jordan, 2011, pp. 32ss.) ${ }^{15}$. Tal instrumento, por sí solo, resulta menos explícito que en la legislación chilena en lo relativo a cuándo se entiende que una empresa ha adoptado tal clase de procedimientos. Sin embargo, en su sección 9 manda al Ministerio de Justicia a publicar una guía sobre los procedimientos que las empresas pueden adoptar para la prevención del soborno (Ministry of Justice (UK), 2011). En esta guía se desarrollan seis principios que debe tener en consideración toda empresa al implementar sus procedimientos para la prevención del soborno. El primero de estos, que es el que interesa acá, indica que tales medidas deben responder a la realidad de la organización en particular, es decir, a los riesgos que enfrenta y la naturaleza, tamaño y complejidad de su actividad comercial ${ }^{16}$.

A su vez, se indica que tales procedimientos deben ser entendibles, aplicables en la práctica, accesibles a sus destinatarios, implementados en forma efectiva y obligatoria para sus asociados. En forma similar a lo que sucede en nuestro ámbito, se reconoce que un sistema de gestión debe partir necesariamente con la identificación y evaluación de los riesgos específicos que enfrenta la empresa en particular (lo que se conoce como risk assessment) y la adopción de respuestas para contrarrestar los mismos y supervisar su efectiva ejecución (Ministry of Justice (UK), 2011, p. 21). Asimismo, tal instrumento otorga algunas directrices fundamentales para la identificación y evaluación de riesgos, como, por ejemplo, su vínculo con la naturaleza y complejidad del negocio, o la relevancia de observar en detalle las actividades o procesos que desarrollan cada uno de sus asociados.

Junto a lo anterior, la guía para la prevención del soborno del Reino Unido sugiere tener en consideración los siguientes riesgos de naturaleza externa (Ministry of Justice (UK), 2011, p. 26), es decir, aquellos que surgirían específicamente de la actividad desarrollada y el lugar en el que se realiza. Para tales efectos, distingue cinco categorías de carácter amplio: el «riesgo país» (country risk), a través del cual se indica que una actividad económica especialmente riesgosa es aquella efectuada en un país con un alto nivel de percepción de corrupción, así como carente de legislación específica en el área o con una escasa promoción de trasparencia por parte de su comunidad. En segundo lugar, indica que se debe tener en consideración el «riesgo sectorial» (sectoral risk),

15 Como explican Bean y MacGuidwin, es la empresa la que debe probar, ante la acusación, que se habían establecido tales procedimientos en forma previa a la comisión del delito $(2013$, p. 88$)$.

16 Los principios 2 al 6 deben entenderse como un refuerzo a las exigencias contenidas en el primer principio relativo a la identificación y evaluación del riesgo, la adopción de respuestas y el monitoreo de las mismas. Así, se expresa que un sistema de prevención del soborno requiere necesariamente del compromiso de la alta dirección (top-level commitment), de una adecuada evaluación de sus riesgos (risk assessment), de la adopción del procedimiento de debido cuidado para operaciones estratégicas (due diligence), de los mecanismos adecuados de capacitación e información (communication, including training) y de la supervisión y monitoreo de la ejecución de los procedimientos implementados (monitoring and review). 
es decir, tener en cuenta que ciertos sectores de la industria son más riesgosos que otros como, por ejemplo, el sector extractivo o el de la infraestructura a gran escala. En tercer lugar, se considera el «riesgo transaccional» (transaction risk), a través del cual se refleja que ciertas transacciones económicas conllevan mayor riesgo que otras, dentro de las que destacan las donaciones, contribuciones políticas, la obtención de permisos o licencias, o las relacionadas con la contratación pública. Luego, en cuarto lugar, describe una serie de riesgos vinculados a las «oportunidades del negocio» (business opportunity risk), ya que ciertas condiciones de este, como, por ejemplo, que sean contratos de un gran valor o que involucren a varios contratistas o intermediarios, así como aquellos que no se tranzan a un valor de mercado, o aquellos que no presenten objetivos legítimos aparentes, pueden implicar mayores niveles de riesgo. Por último, en quinto lugar, se debe analizar el «riesgo asociado a los socios del negocio» (business partnership risk), que especifica qué clase de relaciones comerciales conllevarían mayores riesgos, como, por ejemplo, el uso de intermediarios para relacionarse con funcionarios públicos extranjeros o con personas expuestas políticamente.

De acuerdo con las recomendaciones efectuadas por esta guía, la empresa no solo debe tener en cuenta tales factores externos, sino también evaluar en qué medida su estructura u organización interna pudiera acrecentar el nivel de comisión de tal delito. Así, por ejemplo, se debiera considerar las falencias relativas a la capacitación o entrenamiento de los integrantes de la organización, o una cultura de promoción y bonificación que pudiera recompensar la toma excesiva de riesgos, o una falta de claridad en las políticas y procedimientos que impliquen gastos de hospitalidad, de publicidad o de promoción de sus productos, así como las relativas a contribuciones, aportes políticos o de beneficencia. Por otra parte, se debe atender a una eventual falencia en los controles financieros y, por último, determinar si la alta dirección ha prohibido enfáticamente la realización de actos de corrupción (Ministry of Justice [UK], 2011, p. 26).

Por otra parte, en la guía del Reino Unido analizada, se señala que para la prevención de este delito se deben adoptar no solo políticas específicas, sino también procedimientos diseñados para mitigar los riesgos identificados, como también para prevenir conductas deliberadamente contrarias a la ética que puedan cometer sus asociados - esto es, medidas para detectar incumplimientos intencionales-. Dentro de los procedimientos sugeridos, destacan aquellos vinculados con el establecimiento de canales de denuncia (whistle blowing procedures), medidas disciplinarias, controles de los recursos financieros de la entidad, entrenamiento y capacitación, así como la adopción de procedimientos 
de monitoreo, revisión y evaluación de tales medidas ${ }^{17}$. Cabe destacar, a su vez, que este instrumento otorga especial relevancia al principio de «debida diligencia» (due diligence), cuyo propósito es incentivar que la organización adopte medidas, principalmente relacionadas con la obtención de información relevante, para disminuir que asociados que efectúen un servicio para la organización puedan cometer actos de corrupción en su nombre, así como evaluar los niveles de riesgo asociados a procesos de fusión o adquisición de nuevas compañías ${ }^{18}$. Por último, a través de este principio se recomienda que la empresa adopte las medidas necesarias para contrarrestar los riesgos asociados a la captación o incorporación de nuevo personal que efectúe labores estratégicas.

Uno de los principales aspectos que destaca de esta guía es el hecho de que se reconozca que tales medidas asociadas a la prevención de la corrupción o soborno deben entenderse como parte de un proceso general e integral de la organización dirigido a gestionar todos sus riesgos (Bleker \& Hortensius, 2014, p. 10) ${ }^{19}$. Así, si se observan los componentes de la gestión del riesgo propuestos por el Committee of Sponsoring Organizations of the Treadway Commission (COSO), se puede constatar en forma evidente tal relación.

Como se podrá apreciar, la prevención de la corrupción por las personas jurídicas supone considerar una serie de elementos o componentes mínimos que pueden extraerse de principios generales de la gestión de riesgos corporativos (tales principios se han extraído de COSO, 2017; para los componentes de la gestión del riesgo según COSO, véase Dias, 2017, pp. 76ss.). Estos principios se encuentran organizados en cinco componentes interrelacionados, a saber: a) gobierno corporativo y cultura organizacional, a través del cual se especifica la importancia del establecimiento de un ambiente de control adecuado, ya sea en el ámbito estructural y de control interno (principalmente asignación de responsabilidades de supervisión) o en el refuerzo de una cultura organizacional que potencie valores y un comportamiento ético, así como en la comprensión de los riesgos a los que se enfrenta la organización; b)

17 En lo que respecta a tales medidas de control para la prevención del soborno en el ámbito empresarial, se debe destacar el aporte realizado por la norma ISO 37001 que sugiere la adopción de una serie de controles tanto financieros como no financieros para tales efectos (ISO 37001:2016, anexos 11 y 12).

18 El concepto de "debida diligencia» es usual en el ámbito de los sistemas de gestión antisoborno. Destaca lo dispuesto por la norma ISO 37001, la cual lo entiende como un elemento esencial de tales procesos. Su objeto es evaluar con mayor profundidad el riesgo asociado a determinada clase de operaciones y sirve como un «control específico adicional en la prevención y detección» de tales riesgos. Una de las principales consecuencias asociadas a la realización de la «diligencia debida» es la de decidir acerca de la conveniencia de «posponer, suspender o revisar» determinadas operaciones, proyectos o incluso relaciones con ciertos socios (ISO 37001:2016, anexo 10).

19 Como explican Bleker y Hortensius, basta con observar la estructura de las normas ISO 19600 y 31000 , entre otras, para verificar que las reglas básicas para la gestión del riesgo de incumplimiento legal no son más que una concreción, para tales efectos, de las reglas generales de gestión de riesgos corporativos. 
un establecimiento estratégico de objetivos mediante el cual se reconoce que todo proceso de gestión de riesgos está vinculado a la definición de cuáles son los objetivos que persigue la entidad y la tolerancia al riesgo que estos admiten; c) el componente de «desempeño» mediante el cual se establece la relevancia de la identificación y evaluación de los riesgos específicos a los que se expone la entidad y se sugiere que estos sean priorizados de acuerdo con su gravedad en el marco de la propia definición interna de la tolerancia al riesgo. Una vez evaluados los riesgos, se deben adoptar las medidas de respuesta de acuerdo con las definiciones previas a las que se haya arribado, es decir, si pretende contrarrestarlos o tolerarlos y en qué medida; d) el componente de la «revisión», de acuerdo con el cual la empresa debe determinar el grado de satisfacción con el que operan los componentes anteriores en la práctica y la necesidad de actualizar o modificar las medidas adoptadas a la luz de cambio de circunstancias; e) el último componente es el de la «información, comunicación y denuncia», el cual indica que un sistema eficiente de gestión de riesgos requiere de un proceso permanente de obtención y retroalimentación de información relevante, tanto de fuentes externas como internas a lo largo de toda la organización.

En suma, la adopción de procedimientos adecuados para la gestión del riesgo de comisión de actos de corrupción supone necesariamente reconocer la relevancia que presenta cada uno de estos elementos: la identificación y evaluación de los riesgos, la adopción de medidas de respuesta frente a estos, el monitoreo o supervisión de la ejecución de las mismas, la actualización de estas, una cultura organizacional que potencie el cumplimiento de reglas al interior de la empresa y, especialmente, un control interno que evite su incumplimiento (García, 2016, p. 231).

Por tal motivo, se entiende que organismos especializados en la gestión de riesgos asociados al incumplimiento de la ley (es decir, al área «compliance» $)^{20}$ reconozcan, como punto de partida, la relevancia de los elementos recientemente descritos. Al respecto, no puede dejar de considerarse la norma ISO 19600, calificada como el primer estándar internacional sobre compliance, a través del cual se ofrecen una serie de directrices que debe contener un sistema de cumplimiento para ser considerado como efectivo (Casanovas, 2017,p. 44). Como se señaló con anterioridad, y en sintonía con los principios generales de la gestión del

20 En este sentido, la literatura ha entendido que el área de compliance implica dos grandes focos de atención. En primer lugar, se propone como objetivo la prevención de contravenciones a la ley en el marco de la organización corporativa. Como segundo punto, el compliance conlleva también implicancias normativas, tanto en lo que respecta al seguimiento de normas externas como a la generación de reglas internas dentro de la compañía (Haugh, 2016, pp. 1220-1222). En este sentido, se ha entendido que estas normas vienen a llenar el vacío que dejan los estatutos reguladores y formales (p. 1222), siendo considerado por algunos como un aspecto que va más allá de la aplicación de reglas, generando de esta manera una verdadera cultura organizacional que promueva el cumplimiento de la ley (Griffith, 2015, p. 2093). 
riesgo (contenidos en la norma ISO 31000), este instrumento reconoce como componente central para la implementación de un sistema de cumplimiento el refuerzo de la cultura organizacional (Makowicz, 2015, p. 38) y la identificación y evaluación del riesgo, así como la adopción de medidas de respuesta al mismo, supervisión y actualización del sistema en particular (Bleker \& Hortensius, 2014, p. 6).

Para los efectos de este artículo, resulta especialmente relevante la normativa técnica asociada específicamente a la prevención de la corrupción. En este ámbito, no puede dejar de considerarse, entre otros aspectos, lo sugerido por la normativa ISO 37001. El objeto de esta norma técnica es justamente ofrecer criterios para que diversas organizaciones implementen lo que denomina un «sistema de gestión antisoborno» (ISO 37001:2016, punto 4.4), donde nuevamente se refleja la aproximación basada en la gestión de riesgos ya descrita.

En forma similar a la guía del Reino Unido, analizada con anterioridad, la citada norma ISO da cuenta de una serie de factores de riesgos asociados al contexto de la organización - tanto internos como externosque deben ser considerados al identificar y evaluar la probabilidad de ocurrencia de tal clase de conducta delictiva. Dentro de los elementos externos no solo sugiere examinar «la naturaleza y frecuencia de las interacciones con los funcionarios públicos nacionales o extranjeros que pueden suponer un riesgo para el soborno» (ISO 37001:2016, anexo 4.1), sino que advierte que uno de los pasos más relevantes para evaluar los riesgos asociados al contexto externo estaría dado por el examen de la regulación o normativa vigente del lugar donde se efectúa el negocio, con el objeto de determinar, por ejemplo, las limitaciones relativas a la entrega de regalos o utilización de agentes en el marco de una interacción con un funcionario público ${ }^{21}$.

Como se ha señalado anteriormente, del análisis de tal normativa técnica se desprende que una de las principales preocupaciones que motivan a sus emisores es la detección de factores que deben ser considerados al instaurar sistemas de prevención, ya que, de acuerdo con la experiencia

21 En esta área destacan no solo la norma técnica previamente señalada, ya que no puede dejar de mencionarse, entre otras, la norma BS 10500, cuyo objeto es detallar los componentes de un sistema de gestión antisoborno (British Standards Institution [BSI], 2011). Asimismo, Transparency International UK ha elaborado el documento «The 2010 UK Bribery Act Adequate Procedures» (Wilkinson, 2010). En cuanto a las directrices dictadas por el sector público en el ámbito comparado, destaca también el documento emanado de la Criminal Division of the U.S. Department of Justice y la Enforcement Division of the U.S. Securities and Exchange Commission, titulado «A Resource Guide to the U.S. Foreign Corrupt Practices Act» (2012). En este documento se otorga una serie de recomendaciones para la adaptación de un programa de cumplimiento corporativo vinculado a la prevención de la corrupción. A su vez, debe ser considerado el instrumento que emana de la OECD, "Anti-Corruption Ethics and Compliance Handbook for Business» (2013a), así como el «Programa anticorrupción de ética y cumplimiento para las empresas: guía práctica», emanado de las Naciones Unidas (2013). Todos estos instrumentos tienen en común la aproximación basada al riesgo de soborno ya descrita y que reconoce que sus componentes son fundamentales para la prevención de la corrupción por la empresa. 
en este ámbito, la omisión de cualquiera de estos hace previsible que pueda cometerse un delito en el desarrollo de la actividad económica de la que se trate. Así, se colige que la entidad no solo debe identificar los procesos riesgosos — por ejemplo, aquellos que implican cualquier clase de interacción con un funcionario público — sino que, además, deben ser considerados diversos factores, tanto externos como internos, que podrían incidir en que en tales procesos se termine cometiendo un delito. A este aspecto le asignaremos la mayor relevancia, ya que solo adquieren sentido el resto de los elementos de la gestión del riesgo si se han identificado y evaluado correctamente las posibilidades de que en el desarrollo de la actividad de la empresa se cometa un delito de cohecho.

De lo que se trata acá es, entre otros aspectos, de evaluar la probabilidad de que, en el contexto de tales actividades, uno de los integrantes pueda cometer un delito ${ }^{22}$. Así debe ser entendido justamente lo dispuesto en la legislación chilena por la letra a del numeral 3 del artículo 4 de la ley 20393, que señala que la persona jurídica deberá identificar las «actividades o procesos [...] habituales o esporádicos, en cuyo contexto se genere o incremente el riesgo de comisión» de un delito de cohecho. Como hemos podido observar a partir de la descripción de la normativa técnica en particular, dentro de este contexto destacan los siguientes factores externos: el lugar donde se desarrolla el negocio, la clase de operaciones de la que se trate, la forma como se realiza, etcétera; y, entre los factores internos: el nivel de capacitación y conocimiento que presentan los integrantes de la empresa respecto del delito en particular, la sujeción a valores o cultura organizacional, la presencia de incentivos que pudieran fomentar el incumplimiento de la ley, las falencias vinculadas al control interno, etcétera.

La capacidad de poder evaluar el contexto donde se comete el delito, es decir, el proceso en particular expuesto al riesgo, supone conocer una serie de factores que podrían incidir en que en el marco del desarrollo de la actividad de la que se trate se termine incumpliendo la ley. La pregunta que parece pertinente es determinar qué clase de factores debieran ser conocidos y considerados por la persona jurídica al diseñar su sistema de prevención de delitos. Para tales efectos, resulta imprescindible analizar la literatura especializada, proveniente principalmente de la criminología, dedicada a explicar y detectar los factores de riesgo que la empresa debiera contrarrestar ${ }^{23}$. Como se podrá apreciar a continuación,

22 En estricto rigor, tal evaluación supone determinar no solo la probabilidad de comisión del delito, sino el impacto que el mismo podría generar en la organización (Casanovas, 2017, p. 156). Con todo, como el impacto puede ser medido en atención a la eventual pena que arriesga la entidad -entre otros aspectos-, resulta menos problemático que el de la probabilidad de ocurrencia. Por tal motivo, nos abocaremos exclusivamente a este último aspecto.

23 Como señala Piña, para el adecuado diseño de un sistema de prevención de delitos «es imprescindible considerar también las características criminológicas de la actividad corporativa» (2012, p. 5). En un sentido similar se expresa García al sostener que «el análisis de riesgos de infracción penal es una

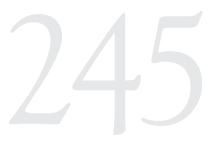

EL DEBER DE GESTIÓN DEL RIESGO DE CORRUPCIÓN EN LA EMPRESA EMANADO DE LA LEY 20393 DE CHILE: ESPECIAL REFERENCIA A LAS EXIGENCIAS DE IDENTIFICACIÓN Y EVALUACIÓN DE RIESGO

THE DUTYTO MANAGETHE RISK OF CORRUPTION IN THE COMPANY EMANATED FROM CHILEAN LAW 20393: SPECIAL REFERENCETOTHE IDENTIFICATION AND RISK ASSESSMENT REQUIREMENTS 
y en absoluta sintonía con los principios generales de la gestión de riesgos de incumplimiento de la ley ya abordados, estos factores pueden tener distinta naturaleza. Por un lado, pueden estar vinculados a factores estructurales de la persona jurídica, asociados principalmente a la idea de que realizar una actividad económica en una organización empresarial de cierta complejidad puede facilitar la comisión de delitos en el sentido de aumentar la oportunidad de comisión, así como dificultar su detección; y a factores que miran a la cultura organizacional en particular y a la posibilidad de que la misma incentive la comisión de conductas delictivas.

\section{FACTORES DE RIESGO CRIMINÓGENOS ASOCIADOS A LA CORRUPCIÓN PROPIOS DE LA ACTIVIDAD EMPRESARIAL}

\section{IV.1. Oportunidades delictivas en contextos organizacionales}

Partiremos de la base de que la comisión de delitos corporativos acaece dentro de un contexto organizacional, donde, si bien las personas se desempeñan de manera autónoma, dicho trabajo se encuentra orientado por el interés común de un equipo (Simpson, 2002, p. 8). En el ámbito de la criminología se han desarrollado distintas corrientes teóricas para explicar las causas de la comisión de estos delitos, usualmente denominados «delitos de cuello blanco» y cometidos en el ámbito corporativo. Los lineamientos clásicos que han encontrado cabida en esta materia corresponden a la teoría de la asociación diferencial, teoría de la anomia, teoría del control, teoría de la elección racional, teorías integradoras y perspectivas desde la teoría de la oportunidad (Benson \& Simpson, 2015, pp. 71ss.).

Ahora, dado que este acápite centra su atención en la comisión de tal clase de delitos, por sobre la caracterización de quienes cometen estos ilícitos, se identificarán los factores de riesgo vinculados a la oportunidad de cometer delitos corporativos, considerando las particularidades que estos revisten. Tal como lo ha sostenido la literatura especializada, saber por qué las personas se comportan de una u otra manera es distinto a explicar por qué un evento - en este caso un delito corporativoacaece en un momento y espacio determinado (Benson \& Simpson, 2015, p. 95).

En concordancia con lo anterior, es posible apuntar que la organización, tanto por su estructura como por su cultura, genera oportunidades para que los actores organizacionales participen en la comisión de delitos (Simpson \& Piquero, 2002, p. 511), siempre y cuando el actor 
tenga conciencia de dicha posibilidad (Coleman, 1987, p. 409). Bajo la perspectiva ofrecida por la teoría de oportunidad, es posible comprender las leyes y regulaciones como mecanismos que pueden ser utilizados para moldear y controlar las oportunidades para la comisión de delitos empresariales (Benson \& Simpson, 2015, p. 247).

La teoría de la oportunidad encuentra plena concordancia con la corriente que analiza la disuasión desde la prevención situacional del delito. Bajo esta corriente, el punto central radica en determinar cómo los potenciales infractores evalúan los costos y beneficios asociados a una estructura particular de oportunidad, y qué pasos pueden ser adoptados para transformar tanto los riesgos como los beneficios asociados a cada oportunidad (Benson \& Madensen, 2007, p. 623). Para ello, una adecuada aproximación a la prevención situacional del delito parte de las siguientes consideraciones: (i) debe enfocarse en delitos específicos, identificando cuáles son las formas delictivas en particular que se busca prevenir; (ii) la forma más efectiva de cambiar la estructura de una oportunidad delictiva es mediante la intervención del ambiente; y (iii) las intervenciones en el ambiente tienen por objeto cambiar la percepción asociada a los costos y beneficios relativos a la comisión de un delito en particular, disuadiendo a eventuales infractores (Benson \& Madensen, 2007, pp. 614-616).

Con todo, la teoría de la prevención situacional del delito ha reconocido una serie de características particulares de los delitos de cuello blanco que serían relevantes al momento de analizar este fenómeno en concreto. De esta manera, se ha sostenido que el estudio del tópico de tales delitos debe partir de las siguientes consideraciones (Benson \& Madensen, 2007, pp. 616-617):

- El infractor posee un acceso privilegiado a la víctima o a su objetivo producto del cargo que ostenta por su trabajo.

- El infractor recurre al engaño u ocultamiento para disimular la existencia del delito y las consecuencias de este, tanto para las víctimas como para el aparato persecutor.

- En esta clase de delitos puede resultar sumamente complejo determinar la intención real del autor, ya que el estado mental de este no podría ser fácilmente desprendido de sus acciones. Por eso se ha señalado que el infractor poseería un estado mental ambiguo al momento de cometer el delito.

- El infractor puede encontrarse físicamente alejado o separado de las víctimas al momento de cometer el delito.

El reconocimiento de estas características específicas resulta fundamental para la detección de focos de riesgo vinculados a la comisión de delitos 
dentro de la empresa. Desde la teoría de la prevención situacional, se han establecido cinco focos en los cuales resulta necesario concentrar los esfuerzos para reducir las oportunidades asociadas a la comisión de estos delitos (Benson \& Madensen, 2007, pp. 617-623).

1. Incrementar los esfuerzos requeridos para poder perpetrar el delito: como se ha destacado recientemente, el infractor en los delitos de cuello blanco tiene un acceso privilegiado o puede estar físicamente separado de la víctima, por lo que, para el establecimiento de barreras u obstáculos para su prevención, se debe tener en cuenta las particularidades de los mismos. En este sentido, la literatura ha sugerido que en este punto se debe aumentar los requisitos y certificaciones para ocupar determinados cargos, aumentando así las barreras para definir qué personas se encuentran capacitadas para trabajar en dicho puesto (Benson \& Madensen, 2007, p. 618) ${ }^{24}$.

2. Elevar el riesgo de detección de delitos: para un adecuado refuerzo del riesgo de detección, es necesario tener en cuenta que en los delitos de cuello blanco el infractor busca - ya sea mediante engaño u ocultación - esconder la verdadera naturaleza de sus actos. En consecuencia, la vigilancia debe apuntar a identificar los actos que ostentan una máscara de aparente legitimidad, pero que en realidad son actos ilícitos (Benson \& Madensen, 2007, p. 619). Por lo mismo, resulta fundamental la mayor relevancia como disuasivo que se le otorga a la posibilidad de ser descubierto, más allá de pretender conseguir los mismos objetivos por medio del establecimiento de sanciones más severas (Benson \& Madensen, 2007, p. 619; Smith, Simpson \& Huang, 2007, p. 656) ${ }^{25}$. Al respecto, la literatura sostiene que el riesgo de detección puede ser incrementado de varias formas, dentro de las que destacan el fortalecimiento de la vigilancia formal y la reducción del anonimato. En general, el incremento del riesgo de la detección, a través de la adopción de sistemas de registro de información — por ejemplo, manutención de correos electrónicos y otros documentos- dificultaría la manutención del estado mental ambiguo descrito con anterioridad, evitando así que los infractores puedan excusarse aduciendo que solo se trató de un error o que no sabían del tema (Benson \& Madensen, 2007, pp. 619-620).

24 Por lo mismo, se entiende que normativa técnica especializada en la prevención de la corrupción, como la norma ISO 37001, le otorgue relevancia a los procesos de obtención de información para el reclutamiento de nuevo personal que ocupe cargos estratégicos (ISO 37001:2016, anexo 8.1).

25 Se entiende así la importancia de la adopción de sistemas de denuncia y de sanciones internas dentro de cualquier sistema de prevención de delitos, como lo exige la letra d del numeral 3 del artículo 4 de la ley 20393. En relación con tales procedimientos, véase Artaza (2013b, pp. 563-565). 
3. Reducir las recompensas asociadas al delito: en el ámbito de la prevención de delitos corporativos resulta fundamental evaluar los sistemas de bonificación y promoción interna con el objeto de evitar cualquier tipo de incentivo al incumplimiento de la regulación que considere exclusivamente los resultados finales de la gestión y no los medios (al respecto, específicamente para la prevención de fraudes en el ámbito médico, véase Benson \& Madensen, 2007, p. 621).

4. Reducir cualquier situación que provoque el delito: debido a la complejidad que revisten una gran fracción de los delitos de cuello blanco, la comisión de estos requiere una planificación por parte del infractor. Por lo mismo, y solo a modo de ejemplo, se ha señalado que limitar las posibilidades de imitación de formas de comisión de delitos anteriores resultaría de gran ayuda para reducir la oportunidad de comisión, por ejemplo, a través de la negativa a publicitar los métodos utilizados por quienes se han involucrado en asuntos fraudulentos (Benson \& Madensen, 2007, p. 622).

5. Hacer más difícil justificar o excusarse del delito: este último foco tiene como centro de atención el proceso de racionalización que llevan a cabo los sujetos dentro de la empresa para cometer delitos. $\mathrm{Al}$ respecto, se busca que la eliminación de excusas disminuya la capacidad de los delincuentes de neutralizar sus sentimientos de culpa o vergüenza. Las justificaciones pueden ser eliminadas mediante el establecimiento de reglas, instrucciones, o alterando la conciencia de los individuos (Benson \& Madensen, 2007, p. 622).

La variedad de focos de atención permite constatar las múltiples dimensiones de la evaluación de la perpetración de un delito. De esta manera, la toma de decisiones implica una evaluación de los costos y beneficios asociados a la comisión de la actividad ilegal, los pros y contras asociados a una oportunidad, ya sea tanto en sus dimensiones económicas como no pecuniarias (Simpson, Gibbs, Rorie, Slocum \& Cohen, 2013, p. 233).

En conexión con el tópico de la gestión de riesgos recientemente abordada, se comprenden entonces las razones por las que se coloca un fuerte acento en el fortalecimiento del control interno de las organizaciones, ya sea en lo que respecta a su estructura y adopción de medidas de supervisión o vigilancia, o el control financiero, o la adecuada asignación de responsabilidades, entre otras. La empresa debe identificar necesariamente aquellas falencias en su control interno que podrían aumentar la oportunidad del delito y adoptar de forma consecutiva medidas para contrarrestarlas. En definitiva, el desarrollo

EL DEBER DE GESTIÓN DEL RIESGO DE CORRUPCIÓN EN LA EMPRESA EMANADO DE LA LEY 20393 DE CHILE: ESPECIAL REFERENCIA A LAS EXIGENCIAS DE IDENTIFICACIÓN Y EVALUACIÓN DE RIESGO

THE DUTYTO MANAGETHE RISK OF CORRUPTION IN THE COMPANY EMANATED FROM CHILEAN LAW 20393: SPECIAL REFERENCETOTHE IDENTIFICATION AND RISK ASSESSMENT REQUIREMENTS 
de una actividad económica en el marco de una organización compleja no controlada o supervisada adecuadamente resulta por sí mismo un riesgo y, en este sentido, resulta exigible para la empresa contrarrestarlo a través de la adopción de medidas contenidas en la normativa técnica respectiva ${ }^{26}$.

\section{IV.2. Técnicas de neutralización y cultura empresarial}

Uno de los hallazgos más interesantes que ha apuntado la literatura en esta área es que los individuos no tienden a verse a sí mismos bajo la etiqueta de corruptos (Anand, Ashforth \& Joshi, 2004, p. 40) o como criminales (Cressey, 1965, p. 12). Así, los sujetos evitan la utilización del término corrupción mediante el empleo de tácticas de racionalización ${ }^{27}$ que les permiten entender sus actos corruptos como si fuesen normales y aceptables en el marco de las actividades propias del rubro de los negocios (Anand et al., 2004, p. 41).

Parte de las raíces de la teoría de la racionalización se encuentra en Cressey, quien, partiendo de los postulados de Sutherland sobre el delito de cuello blanco, apuntó que uno de los requisitos necesarios para que un sujeto cometa un delito de malversación - junto a la necesidad financiera y la violación de la confianza que otorga la posición laboral — es la verbalización. Esta última exigencia corresponde al quid del asunto, donde el delincuente debe tener la habilidad para describir el acto de malversación a través de palabras que no lo conflictúen con la imagen que tiene de sí mismo como una persona confiable (1965, pp. 13-14) ${ }^{28}$. Esta práctica posteriormente fue denominada «técnicas de neutralización» (Sykes \& Matza, 1957, p. 667).

Uno de los aspectos más relevantes de las técnicas de neutralización es el hecho de que estas prácticas pueden operar tanto ex ante como ex post con respecto al acto delictivo. Tempranamente, Cressey apuntó que la verbalización corresponde a un vocabulario de motivos que no es creado en el mismo instante en que se comete el acto, sino que más bien proviene de un catálogo disponible con anterioridad (1965, p. 14). En este sentido, las racionalizaciones son creadas con anterioridad a la comisión del acto, permitiendo la perpetración de los delitos (Haugh, 2015, p. 1215).

$26 \mathrm{Al}$ respecto, resultan especialmente útiles las sugerencias de control efectuadas por la norma ISO 37001 , la cual destaca la planificación y control operacional, la debida diligencia, los controles financieros y no financieros.

27 Este texto utiliza los términos racionalización y neutralización como sinónimos, en concordancia con lo ya apuntado por Haugh (2015, p. 1214).

28 Cressey ejemplifica lo anterior poniendo el caso de un empleado honesto que actualmente tiene problemas financieros. Este individuo de ninguna manera aceptaría la oración expresada en términos de «robarle el dinero a su jefe», ya que vería con horror ese tipo de conductas. Lo anterior, sin embargo, cambia radicalmente si el individuo sostiene que «tomará prestado el dinero", lo que le permite mantener su calidad de ciudadano honesto frente a la sociedad (1965, pp. 13-14). 
El nexo entre cultura organizacional y prácticas de racionalización no es menor. Si las racionalizaciones se convierten en un recurso compartido dentro de la cultura organizacional, aquello puede conformar los cimientos para el devenir de una forma de hacer las cosas cuya expresión anglosajona se resume en «business as usual-the way things work» (Anand et al., 2004, p. 41). Al respecto, los factores culturales pueden servir como justificación o para la mitigación de responsabilidades en atención a las circunstancias (Shover \& Hochstetler, 2002, p. 14). Por eso, el reconocimiento de una cultura de cumplimiento resulta fundamental para efectos de comprender el contexto donde acontecen los delitos empresariales y, en particular, los ilícitos cometidos en beneficio de la empresa.

Con todo, es importante destacar que el fenómeno de la racionalización por sí solo no basta para mantener vigente las prácticas corruptas con el paso del tiempo. En este sentido, la literatura señala que en cualquier organización la corrupción solo puede subsistir si los sujetos que ingresan al círculo también comienzan a manifestar estos comportamientos (Anand et al., 2004, p. 44). Este fenómeno es conocido como proceso de socialización, mediante el cual las personas que entran en la compañía aceptan las prácticas usuales como normales (2004, p. 40). Mediante su acción conjunta, las prácticas de racionalización y socialización permiten a los sujetos que participan de actividades contrarias a la ética sentirse como individuos éticos y morales, permitiéndoles continuar el desarrollo de sus prácticas sin sentimientos de remordimiento (p. 40 ${ }^{29}$.

Estudios cualitativos han relevado el espíritu de «guerra» que habría dentro de la cultura de organizaciones corruptas. La presión de perder empleos, sumada a la visión de que se está en una verdadera batalla - potenciada por condiciones flexibles del mercado- cambiarían la percepción que tienen los individuos de la corrupción. Esto provoca un cambio en la percepción de los individuos, quienes enfocan su atención en sobrevivir a tal guerra y conservar sus empleos, dejando en un segundo plano las consideraciones morales y éticas (Campbell \& Göritz, 2014, pp. 291-311). Por lo mismo, se comprende que la gestión de riesgos penales también dependa de una adecuada interiorización de valores por parte de los integrantes de la empresa, lo que habitualmente se efectúa a través del establecimiento de límites éticos a la consecución de los objetivos corporativos. De esta manera, resulta de especial relevancia hoy en día la adopción de códigos de ética o de conducta (Navas, 2013, pp. 122-125).

EL DEBER DE GESTIÓN DEL RIESGO DE CORRUPCIÓN EN LA EMPRESA EMANADO DE LA LEY 20393 DE CHILE: ESPECIAL REFERENCIA A LAS EXIGENCIAS DE IDENTIFICACIÓN YEVALUACIÓN DE RIESGO

THE DUTYTO MANAGETHE RISK OF CORRUPTION IN THE COMPANY EMANATED FROM CHILEAN LAW 20393: SPECIAL REFERENCETOTHE IDENTIFICATION AND RISK ASSESSMENT REQUIREMENTS 
Siguiendo la sistematización realizada por Haugh (2016, pp. 1255ss.), las principales técnicas de racionalización, conocidas en la actualidad, serían las siguientes.

1. Negación de la responsabilidad: esta racionalización implica que el criminal define su conducta de forma tal que disminuye su responsabilidad, lo que generalmente es efectuado a través de la excusa de que su comportamiento fue accidental, o que se debe a presiones que están fuera de su control (Maruna \& Copes, 2005, p. 232). En este sentido, es posible encontrar justificaciones circunstanciales como aquellas que apuntan a explicar la conducta como consecuencia de que no habrían tenido otra opción —es decir, no habrían podido realmente elegir—, lo cual puede explicarse por estar insertos en un sistema coercitivo, donde abundan las crisis financieras o las presiones de pares (Anand et al., 2004, p. 41). Esta técnica de neutralización no hace sino confirmar que la interpretación de la responsabilidad es una construcción cultural, yendo más allá de ser meras creencias idiosincráticas (Sykes \& Matza, 1957, p. 667). Tal técnica de justificación resulta especialmente relevante en el ámbito de la prevención de la corrupción, sobre todo en lo que atañe a la posibilidad de explicar tales conductas como una consecuencia inevitable de las altas tasas de corrupción del lugar en el que se desarrolla el negocio y que, por tanto, sería la única forma de conseguir los objetivos de la organización (Nieto, 2013, p. 191). Así, por ejemplo, quien sostiene que no es más que una víctima de funcionarios públicos corruptos que solicitan beneficios económicos ilegales para llevar a cabo las funciones propias de su cargo.

2. Negación del daño: la justificación tiene su base en la asunción de que nadie realmente será dañado con la conducta (Maruna \& Copes, 2005, p. 232). Este tipo de racionalización también opera cuando la víctima es una empresa, por lo que se asume que esta se encuentra debidamente asegurada y puede fácilmente recuperar los costos (Anand et al., 2004, p. 42). De esta manera, esta técnica de neutralización permite diferenciar y sostener que no todo acto ilegal es un acto inmoral (Sykes \& Matza, 1957, p. 667).

3. Negación de la víctima: esta racionalización puede adoptar dos formas (Maruna \& Copes, 2005, p. 232). La primera es mediante el entendimiento de que la conducta de la víctima fue inapropiada, por lo que, al final del día, merecería el daño (Maruna \& Copes, 2005, p. 232). La segunda vía es mediante la consideración de que la víctima es difícil de identificar o abstracta (Maruna \& Copes, 2005, p. 232). En este sentido, al 
estar físicamente ausente la víctima, su visibilidad se ve debilitada (Sykes \& Matza, 1957, p. 668).

4. Condenando a los condenadores: esto se realiza cambiando el foco de atención desde las propias conductas a los motivos que habría detrás de otras personas o grupos, como los reguladores y agencias persecutoras (Sykes \& Matza, 1957, p. 668). Esta forma de racionalización puede adoptar el siguiente razonamiento: si la legitimidad de los actores o entidades es cuestionable, también lo son, en consecuencia, los argumentos vertidos por ellos (Anand et al., 2004, p. 43).

5. La apelación a lealtades superiores: esto ocurre cuando un individuo considera más importantes las demandas del grupo al que pertenece que la normativa legal. Es elemental destacar que en este tipo de racionalización es posible que se pase de la neutralización a una evaluación del acto corrupto mediante una ponderación, considerándose que las metas del grupo corporativo son prioritarias frente a las de la sociedad $\mathrm{u}$ otros grupos (Anand et al., 2004, p. 43) ${ }^{30}$.

6. La metáfora de Ledger: la racionalización implica que, pese a que los delincuentes aceptan que han causado daño, neutralizan su comportamiento comparándolo con el buen comportamiento previo que han tenido en la empresa.

7. Reclamo de relativa aceptabilidad: esta técnica se lleva a cabo mediante la comparación de la conducta propia con la de otras personas que han actuado en forma similar.

Las tácticas de racionalización recientemente descritas no son más que herramientas que pueden ser usadas para la justificación de prácticas contra la ética, resultando en desastrosas consecuencias para la sociedad y, eventualmente, para la organización misma (Anand et al., 2004, p. 44). Es importante destacar que los empleos de las racionalizaciones no son uniformes ni excluyentes entre sí, por lo que pueden variar con el paso del tiempo, ser utilizadas en distintos grados y combinadas entre sí (Haugh, 2015, p. 1222).

Si bien las técnicas de racionalización y socialización pueden existir en alguna medida sin algún soporte por parte del ambiente organizacional, existen algunos factores que pueden aumentar significativamente la probabilidad de que aparezcan estos dos procesos (Anand et al., 2004, p. 46). En este marco dinámico, la literatura ha identificado tres focos de relevancia a considerar en el desarrollo de técnicas de racionalización.

EL DEBER DE GESTIÓN DEL RIESGO DE CORRUPCIÓN EN LA EMPRESA EMANADO DE LA LEY 20393 DE CHILE: ESPECIAL REFERENCIA A LAS EXIGENCIAS DE IDENTIFICACIÓN YEVALUACIÓN DE RIESGO

THE DUTYTO MANAGETHE RISK OF CORRUPTION IN THE COMPANY EMANATED FROM CHILEAN LAW 20393: SPECIAL REFERENCETOTHE IDENTIFICATION AND RISK ASSESSMENT REQUIREMENTS 
1. Atracción social y capullo social: un capullo social es una microcultura creada dentro de un grupo, donde las normas se ven desde una perspectiva diversa a la valoración que tiene la sociedad de ellas o, incluso, la propia organización en su conjunto (Anand et al., 2004, p. 46). Los capullos sociales emergen cuando los grupos generan soluciones idiosincráticas a problemas que enfrentan conjuntamente bajo presiones externas (p. 46). En este sentido, los capullos sociales han sido entendidos como parte de una cultura organizacional corrupta cuyo propósito es garantizar el apoyo por parte de los empleados a la comisión de estas conductas (Campbell \& Göritz, 2014, p. 294).

2. Soporte mutuo de racionalización y socialización: los procesos de racionalización se apoyan y refuerzan mutuamente. Así, si un nuevo integrante de la empresa comete su primera conducta corrupta y experimenta una fuerte discordancia con lo que se espera de él por parte de la organización, es probable que lo anterior prevenga que tal conducta continúe. Si, por el contrario, se enfrenta a condiciones culturales que puedan morigerar dicha discordancia, aumentaría la probabilidad de que el acto continúe (Anand et al., 2004, pp. 46-47).

3. Lenguaje eufemístico: el lenguaje eufemístico permite a los individuos cometer actos de corrupción al describir sus actos de forma tal que estos parezcan inofensivos (Anand et al., 2004, p. 47).

En conformidad con la perspectiva de la prevención situacional ya reseñada, se asume que los sujetos son racionales y tienen algún grado de sensibilidad ante los cambios que se producen en el ambiente, provocando un cambio en la consideración de costos y beneficios (Benson \& Madensen, 2007, p. 616). Por tanto, existe la posibilidad de reducir la racionalización y socialización dentro de las empresas mediante la intervención de la cultura ética presente en cada una de ellas. En este sentido, la literatura ha sugerido algunas medidas.

1. Fomentar la conciencia entre los empleados: el entrenamiento de los empleados para evitar prácticas que promuevan la racionalización —como el lenguaje eufemístico y capullos sociales - puede ser una medida a largo plazo para mejorar el clima ético dentro de la corporación (Anand et al., 2004, p. 48). El entrenamiento periódico sobre acciones o decisiones futuras, tomando en consideración la perspectiva de los consumidores, accionistas y otros actores puede ayudar en este tema. Lo anterior ha sido denominado test headline: «iQué pensarían los actores de una organización sobre el acto o decisión tomada si esta fuese reportada en los medios?» (p. 48). 
2. Uso de evaluaciones de rendimiento que vayan más allá de los números: en este sentido, se destaca la necesidad de realizar evaluaciones externas que puedan explorar aspectos que no se midan estrictamente en resultados. Así, por ejemplo, bajo la directriz numérica, el evaluador puede verse impedido de profundizar en el análisis producto de la existencia de un lenguaje eufemístico y capullos sociales. De esta manera, se debe focalizar en investigar cómo se han obtenido los resultados que ostenta la empresa (Anand et al., 2004, p. 49).

3. Alimentar el ambiente ético de la organización: esta medida parte de la base de que, si bien el establecimiento de códigos de ética corresponde a un avance positivo, no es completamente suficiente (Anand et al., 2004, p. 49). En este sentido, un código de ética puede influenciar el comportamiento, el cual debe ser respaldado por las estructuras y políticas organizacionales (p. 49). Así, este soporte organizacional y estructural puede desarrollarse por distintas vías:

a. Establecer mecanismos para que los empleados, ante incertezas o dudas frente al carácter corrupto de sus actos, puedan conversar estos asuntos con un representante independiente de la compañía. Mediante esta medida, resulta posible que los individuos discutan asuntos éticos sin miedo a represalias (Anand et al., 2004, p. 49).

b. La organización debe tener fuertes mecanismos de control de procesos más allá de códigos de ética durante la realización de actividades fundamentales para el funcionamiento de la empresa.

4. La conducta de gerentes como modelos de ética: sin lugar a dudas, las tácticas de racionalización son facilitadas si los individuos que ostentan altos cargos en la empresa incurren en actos en contra de la ética. Por lo mismo, se entiende que una de las conclusiones más relevantes de estudios como el desarrollado por Smith, Simpson y Huang subraye la importancia de legitimar el uso de la ética tanto en la toma de decisiones como en el discurso en torno a los negocios (Smith et al., 2007, p. 656). De lo contrario, la cultura organizacional podría llegar incluso a valorizar la corrupción y diseñar fórmulas para su comisión (Ashforth \& Anand, 2003, p. 9).

\section{CONCLUSIONES}

Como ha quedado de manifiesto, el deber de dirección y supervisión emanado del sistema chileno de atribución de responsabilidad penal

EL DEBER DE GESTIÓN DEL RIESGO DE CORRUPCIÓN EN LA EMPRESA EMANADO DE LA LEY 20393 DE CHILE: ESPECIAL REFERENCIA A LAS EXIGENCIAS DE IDENTIFICACIÓN YEVALUACIÓN DE RIESGO

THE DUTYTO MANAGETHE RISK OF CORRUPTION IN THE COMPANY EMANATED FROM CHILEAN LAW 20393: SPECIAL REFERENCETOTHE IDENTIFICATION AND RISK ASSESSMENT REQUIREMENTS 
corporativa puede ser perfectamente concretado, entre otros, como un deber de gestionar los riesgos de comisión de actos de corrupción generados por el ejercicio de la actividad de la empresa. Tal deber, como se pudo extraer del análisis de la práctica comparada y la normativa técnica respectiva, supone la confluencia del desarrollo de varios componentes diversos que, simplificando en exceso la cuestión, pueden ser asociados a la identificación y evaluación de los riesgos, así como a la adopción de medidas de respuesta frente a estos y la supervisión y actualización de estas. A su vez, se abordó el importante papel que desempeña el refuerzo de una cultura organizacional que promueva el cumplimiento de la ley como un elemento central de la gestión eficiente de tales riesgos.

Unode los elementoscentrales de este deber esidentificar adecuadamente aquellos riesgos que genera la actividad y su posterior evaluación, como paso previo a la adopción de las medidas para poder contrarrestar dichos riesgos de acuerdo con su gravedad e importancia. Para tales efectos, resulta fundamental no solo singularizar aquellos procesos de la entidad o actividades en cuyo contexto se pueda cometer tal delito. Además, y lo que resulta aún más relevante, se debe evaluar la probabilidad de que en el marco del desarrollo de dichas actividades — este es el sentido que se le debiera asignar al término «contexto»— se termine cometiendo tal delito para la consecución o fomento de los intereses de la empresa.

Como se pudo observar a partir de la normativa técnica analizada, al evaluar tal probabilidad, se debía tener en cuenta factores de riesgo tanto internos como externos a la organización. La normativa se ha centrado, principalmente, en la detección de aquellos factores asociados, más bien, al tipo de negocios y al lugar donde se desarrollan, mientras que la criminología especializada en las causas del delito corporativo se ha encargado, en forma prioritaria, del estudio de factores internos a la organización que podrían explicar el surgimiento de actos de corrupción. Estos últimos podrían ser incluidos en dos categorías diversas: por una parte, aquellos relacionados con el aumento de la oportunidad del delito, donde destacan aquellos factores asociados a la facilitación de su comisión debido a que se realizarían en el marco de estructuras organizativas de cierta complejidad que dificultarían su detección o potenciarían la percepción de posible impunidad frente a su ejecución. Por otra parte, se analizaron aquellos factores de riesgo que fomentarían la conducta delictiva a través de la incidencia que podría presentar para los integrantes de la empresa su propia cultura organizacional. Destacan acá los avances presentados por la teoría de la neutralización o la racionalización de la conducta ilícita en el marco de los negocios.

En suma, cada uno de estos factores - tanto externos como internos, ya sea que estén relacionados con el tipo de negocios, con la estructura 
o control interno de la organización o con su propia cultura— podría aumentar la probabilidad de comisión de actos de corrupción en interés de la empresa y, por lo mismo, deben ser necesariamente considerados al evaluar sus propios riesgos, con el objeto de satisfacer el mandato de dirección y supervisión emanado de la ley 20393. En caso de que un integrante de la persona jurídica cometa un delito de cohecho y se detecte que esta no había adoptado medidas para contrarrestar cualquiera de los factores de riesgo ya descritos, se cumpliría al menos una de las exigencias para sostener que tal conducta delictiva es una consecuencia de la omisión de los deberes de dirección y supervisión, en la medida que resultaba previsible para la compañía la incidencia que podrían presentar tales factores en la ejecución de sus procesos o actividades. La otra exigencia (que no ha podido ser abordada acá) para el cumplimiento del requisito de la satisfacción del vínculo con la omisión del deber de gestión es que sea posible para la empresa contrarrestar tales factores a través de la adopción de medidas de cuidado. Para tales efectos, resulta evidente que el análisis también se puede efectuar a través de la aproximación a la gestión de riesgos ya descrita, pero en lo que atañe al componente de la exigencia de adopción de medidas en particular para prevenir conductas delictivas y al análisis de prácticas asentadas en el área del compliance.

REFERENCIAS

Alldridge, P. (2012). The U.K. Bribery Act: The Caffeinated Younger Sibling of the FCPA. Ohio State Law Joumal, 73(5), 1781-1216. Recuperado de http://hdl. handle.net/1811/71562

Anand, V.; Ashforth, B. \& Joshi, M. (2004). Business as Usual: The Acceptance and Perpetuation of Corruption in Organizations. The Academy of Management Executive, 18(2), 39-53. https://doi.org/10.5465/ame.2004.13837437

Artaza, O. (2013a). La empresa como sujeto de imputación de responsabilidad penal: Fundamentos y limites. Madrid: Marcial Pons.

Artaza, O. (2013b). Sistemas de prevención de delitos o programas de cumplimiento: breve descripción de las reglas técnicas de gestión del riesgo empresarial y su utilidad en sede jurídico penal. Politica Criminal, 8(16), 544-573. https://doi.org/10.4067/s0718-33992013000200006

Ashforth, B. \& Anand, V. (2003). The Normalization of Corruption in Organizations. Research in Organizational Behavior, 25, 1-52. https://doi. org/10.1016/s0191-3085(03)25001-2

Balmaceda, G. \& Guerra, R. (2014). Politicas de prevención de delitos en la empresa. Santiago: Legal Publishing-Thomson Reuters.

Bean, B. \& MacGuidwin, E. (2012). Expansive Reach - Useless Guidance: An Introduction to the U.K. Bribery Act 2010. ILSA Journal of International $\mathbb{E}$ 
Comparative Law, 18(2), 323-346. Recuperado de https:/digitalcommons.law. msu.edu/facpubs/496/

Bean, B. \& MacGuidwin, E. (2013). Unscrewing the Inscrutable: The UK Bribery Act 2010. Indiana International \&3 Comparative Law Review, 23(1), 63 109. Recuperado de http://journals.iupui.edu/index.php/iiclr/article/view/17873

Benson, M. \& Madensen, T. (2007). Situational Crime Prevention and White-Collar Crime. En H. Pontell y G. Geis (eds.), International Handbook of White-Collar and Corporate Crime (pp. 609-626). Boston: Springer. https://doi. org/10.1007/978-0-387-34111-8_31

Benson, M. \& Simpson, S. (2015). Understanding White-Collar Crime: An Opportunity Perspective ( $2^{a}$ ed.). Nueva York: Routledge. https://doi. org/10.4324/9780203762363

Bleker, S. \& Hortensius, D. (2014). ISO 19600: The Development of a Global Standard on Compliance Management. Business Compliance, 2, 1-12. Recuperado de http://www.nen.nl/web/file?uuid=81d60dcf$\mathrm{dd} 04-4820-8 \mathrm{ccb}-\mathrm{bb} 685 \mathrm{a} 0786 \mathrm{fc}$ \&owner $=\mathrm{ccdd} 2 \mathrm{a} 27-7 \mathrm{f} 28-43 \mathrm{~b} 1-\mathrm{a} 3 \mathrm{cb}-$ d01e2bf2a56a\&contentid $=171861$

Campbell, J. \& Göritz, A. (2014). Culture Corrupts! A Qualitative Study of Organizational Culture in Corrupt Organizations. Journal of Business Ethics, 120(3), 291-311. https://doi.org/10.1007/s10551-013-1665-7

Carnevali, R. \& Artaza, O. (2016). La naturaleza pluriofensiva y transnacional del fenómeno de la corrupción: desafíos para el derecho penal. Revista Ius et Praxis, 22(2), 53-90. https://doi.org/10.4067/s0718-00122016000200003

Casanovas, A. (2017). Compliance penal normalizado: el estándar UNE 19601. Pamplona: Aenor Ediciones-Thomson Reuters Aranzadi.

Coca, I. (2013). ¿Programas de cumplimiento como forma de autorregulación regulada? En J.M. Silva Sánchez (dir.), Criminalidad de empresa y compliance: prevención y reacciones corporativas (pp. 43-76). Barcelona: Atelier.

Coleman, J. (1987). Toward an Integrated Theory of White-Collar Crime. American Joumal of Sociology, 93(2), 406-439. https://doi.org/10.1086/228750

Cressey, D. (1965). The Respectable Criminal. Trans-action, 2(3), 12-15. https:// doi.org/10.1007/bf03180809

Dias, A. (2017). A more effective audit after COSO ERM 2017 or after ISO 31000:2009? Perspectiva Empresarial, 4(2), 73-82. Recuperado de https://rpe. ceipa.edu.co/index.php/perspectiva-empresarial/article/view/134

Dunst, L.; Diamant, M. \& Kung, T. (2011). Hot Off the Press: Resetting the Global Anti-Corruption Thermostat to the UK Bribery Act. Business Law International, 12 (3), 257-292. Recuperado de https://heinonline.org/HOL/P?h=hein.journals/ blawintnl12\&i=265 
García, P. (2016). Las políticas anticorrupción en la empresa. Revista de Derecho de la Pontificia Universidad Católica de Valparaíso, 67, 219-244. https://doi. org/10.4067/s0718-68512016000200007

Gómez-Jara, C. (2012). Fundamentos de la responsabilidad penal de las personas jurídicas. En M. Bajo, B. Feijoo \& C. Gómez-Jara (dirs.), Tratado de responsabilidad penal de las personas jurídicas (pp. 109-133). Navarra: Civitas Aranzadi.

Griffith, S. (2015). Corporate Governance in an Era of Compliance. William E) Mary Law Review, 57(6), 2075-2140. Recuperado de https://ssrn.com/ abstract $=2766661$

Haugh, T. (2015). Overcriminalization's New Harm Paradigm. Vanderbilt Law Review, 68(5), 1191-1241. Recuperado de https://www.vanderbiltlawreview.org/ wp-content/uploads/sites/89/2015/10/Overcriminalization2.pdf

Haugh, T. (2016). The Criminalization of Compliance. Notre Dame Law Review, 92(3), 1215-1270. Recuperado de https:/scholarship.law.nd.edu/ndlr/vol92/ iss $3 / 5$

Hernández, H. (2010). La introducción de la responsabilidad penal de las personas jurídicas en Chile. Política Criminal, 5(9), 207-236. https://doi.org/10.4067/s071833992010000100005

Hunter, S. (2011). A Comparative Analysis of the Foreign Corrupt Practices Act and the U.K. Bribery Act, and the Practical Implications of both on International Business. ILSA Journal of International Eु Comparative Law, 18(1), 89-113. Recuperado de https://nsuworks.nova.edu/ilsajournal/vol18/iss1/4

Jordan, J. (2011). The Adequate Procedures Defense under the UK Bribery Act: A British Idea for the Foreign Corrupt Practices Act. Stanford Journal of Law, Business $\mathcal{E}$ Finance, 17(1), 25-66. Recuperado de https:/heinonline.org/HOL/ $\mathrm{P} ? \mathrm{~h}=$ hein.journals/stabf $17 \& \mathrm{i}=27$

Jordan, J. (2012). The Need for a Comprehensive International Foreign Bribery Compliance Program, Covering A to Z, in an Expanding Global Anti-Bribery Environment. Penn State Law Review, 117(1), 89-137. Recuperado de http:// www.pennstatelawreview.org/print-issues/the-need-for-a-comprehensiveinternational-foreign-bribery-compliance-program/

Koehler, M. (2010). The Foreign Corrupt Practices Act in the Ultimate Year of Its Decade of Resurgence. Indiana Law Review, 43(2), 389-421. https://doi. org/10.18060/4041

Koehler, M. (2012). Revisiting a Foreign Corrupt Practices Act Compliance Defense. Wisconsin Law Review, 2, 609-659. Recuperado de https://ssrn.com/ abstract $=1982656$

Lu, T. (2013). The «Obtaining Or Retaining Business» Requirement: Breathing New Life into the Business Nexus Provision of the FCPA. Fordham Joumal of Corporate EF Financial Law, 18(3), 729-750. Recuperado de https://irlawnet. fordham.edu/jcfl/voll8/iss3/10 
Mackenzie, S. \& Yates, D. (2016). Collectors on Illicit Collecting: Higher Loyalties and Other Techniques of Neutralization in the Unlawful Collecting of Rare and Precious Orchids and Antiquities. Theoretical Criminology, 20(3), 340357. https://doi.org/10.1177/1362480615607625

Makowicz, B. (2015). The Global Compliance Management System: A Practical Approach to Compliance across Cultures. Business Compliance, 6, 35-44. Recuperado de http://compliance-academia.de/wp-content/uploads/2015/12/ Prof_Makowicz_Business-Compliance.pdf

Manacorda, S. (2014). Towards an Anti-Bribery Compliance Model: Methods and Strategies for a «Hybrid Normativity». En S. Manacorda, F. Centonze \& G. Forti (eds.), Preventing Corporate Corruption: The Anti-Bribery Compliance Model (pp. 3-30). Cham: Springer. https://doi.org/10.1007/978-3-319-04480-4_1

Mañalich, J. (2011). Organización delictiva: bases para su elaboración dogmática en el derecho penal chileno. Revista Chilena de Derecho, 38(2), 279-310. https:// doi.org/10.4067/s0718-34372011000200005

Maruna, S. \& Copes, H. (2005). What Have We Learned from Five Decades of Neutralization Research? Crime and Justice, 32, 221-320. https://doi. org/10.1086/655355

Mukwiri, J. (2015). British law on corporate bribery. Joumal of Financial Crime, 22(1), 16-27. https://doi.org/10.1108/jfc-12-2013-0072

Muravska, J. (2014). How to Bribe: A Typology of Bribe-Paying and How to Stop It. Londres: Transparency International UK. Recuperado de http://www. transparency.org.uk/publications/how-to-bribe-a-typology-of-bribe-paying-andhow-to-stop-it/

Navas, I. (2013). Los códigos de conducta y el derecho penal económico. En J.M. Silva Sánchez (dir.), Criminalidad de empresa y compliance: prevención y reacciones corporativas (pp. 111-129). Barcelona: Atelier.

Nieto, A. (2008). Responsabilidad social, gobierno corporativo y autorregulación: sus influencias en el derecho penal de la empresa. Política Criminal, 3(5), 1-18. Recuperado de http://www.politicacriminal.cl/n_05/A_3_5.pdf

Nieto, A. (2013). La privatización de la lucha contra la corrupción. En L. Arroyo $\&$ A. Nieto (dirs.), El derecho penal económico en la era del compliance (pp. 191210). Valencia: Tirant lo Blanch.

Pacini, C. (2012). The Foreign Corrupt Practices Act: Taking a Bite Out of Bribery in International Business Transactions. Fordham Journal of Corporate $\mathbb{E}$ Financial Law, 17(2), 545-589. Recuperado de https://ir.lawnet.fordham.edu/jcfl/ vol17/iss $2 / 5$

Paternoster, R. \& Simpson, S. (1996). Sanction Threats and Appeals to Morality: Testing a Rational Choice Model of Corporate Crime. Law and Society Review, 30(3), 549-584. https://doi.org/10.2307/3054128

Piña, J. (2012). Modelos de prevención de delitos en la empresa. Santiago: Legal Publishing-Thomson Reuters. 
Rose, C. (2012). The UK Bribery Act 2010 and Accompanying Guidance: Belated Implementation of the OECD Anti-Bribery Convention. International and Comparative Law Quarterly, 61, 485-499. https://doi.org/10.1017/ s0020589312000036

Shover, N. \& Hochstetler, A. (2002). Cultural Explanation and Organizational Crime. Crime, Law and Social Change, 37(1), 1-18. https://doi. org/10.1023/a:1013399001934

Simpson, S. (2002). Corporate crime, law, and social control. Cambridge: Cambridge University Press. https://doi.org/10.1017/cbo9780511606281

Simpson, S., Gibbs, C., Rorie, M., Slocum, L. \& Cohen, M. (2013). An Empirical Assessment of Corporate Environmental Crime-Control Strategies. The Journal of Criminal Law and Criminology, 103(1), 231-278. Recuperado de https:// scholarlycommons.law.northwestern.edu/jclc/vol103/iss1/5

Simpson, S. \& Piquero, N. (2002). Low Self-Control, Organizational Theory, and Corporate Crime. Law and Society Review, 36(3), 509-548. https://doi. org/10.2307/1512161

Smith, C., Simpson, S. \& Huang, C. (2007). Why Managers Fail to Do the Right Thing: An Empirical Study of Unethical and Illegal Conduct. Business Ethics Quarterly, 17(4), 633-667. https://doi.org/10.1017/s1052150x00002633

Sykes, G. \& Matza, D. (1957). Techniques of Neutralization: A Theory of Delinquency. American Sociological Review, 22(6), 664-670. https://doi. org/10.2307/2089195

Wilkinson, P. (2010). The 2010 UK Bribery Act Adequate Procedures: Guidance on Good Practices Procedures for Corporate Anti-Bribery Programmes. Londres: Transparency International UK. Recuperado de https://www.transparency.org. uk/publications/adequate-procedures-guidance-to-the-uk-bribery-act-2010/

\section{Jurisprudencia, normativa y otros documentos legales}

British Standards Institution (BSI) (2011). BS 10500:2011 Specification for an anti-bribery management system (ABMS).

Committee of Sponsoring Organizations of the Treadway Commission (COSO) (2017). Enterprise Risk Management: Integrating with the Strategy and Performance. Executive Summary. Recuperado de https://www.coso.org/ Documents/2017-COSO-ERM-Integrating-with-Strategy-and-PerformanceExecutive-Summary.pdf

Criminal Division of the U.S. Department of Justice \& Enforcement Division of the U.S. Securities and Exchange Commission (2012). A Resource Guide to the U.S. Foreign Corrupt Practices Act. Recuperado de https://www.sec.gov/ spotlight/fcpa/fcpa-resource-guide.pdf

ISO (Organización Internacional de Normalización) (2016). ISO 37001:2016 Sistemas de gestión antisoborno - Requisitos con orientación para su uso.

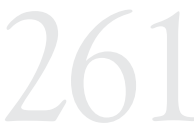

EL DEBER DE GESTIÓN DEL

RIESGO DE

CORRUPCIÓN EN LA

EMPRESA EMANADO

DE LA LEY 20393

DE CHILE: ESPECIAL

REFERENCIA A LAS

EXIGENCIAS DE

IDENTIFICACIÓN

Y EVALUACIÓN DE

RIESGO

THE DUTYTO

MANAGETHE RISK

OF CORRUPTION

IN THE COMPANY

EMANATED FROM

CHILEAN LAW

20393: SPECIAL

REFERENCETOTHE

IDENTIFICATION

AND RISK

ASSESSMENT

REQUIREMENTS 
Ley 20393, Establece la Responsabilidad Penal de las Personas Jurídicas en los Delitos de Lavado de Activos, Financiamiento del Terrorismo y Delitos de Cohecho que Indica, 25 de noviembre de 2009, Diario Oficial [D.O.], 2 de diciembre de 2009 (Chile).

Ministry of Justice (UK) (2011). The Bribery Act 2010: Guidance about Procedures which Relevant Commercial Organisations Can Put into Place to Prevent Persons Associated with Them from Bribing (Section 9 of the Bribery Act 2010). Recuperado de http://www.justice.gov.uk/downloads/legislation/ bribery-act-2010-guidance.pdf

Naciones Unidas (2013). Programa anticorrupción de ética y cumplimiento para las empresas: guía práctica. Nueva York. Recuperado de https://www.unodc.org/ documents/corruption/Publications/2013/13-85255_Ebook.pdf

Organización para la Cooperación y el Desarrollo Económicos (OECD) (2013a). Anti-Corruption Ethics and Compliance Handbook for Business. Recuperado de http://www.oecd.org/corruption/Anti-CorruptionEthicsComplianceHandbo ok.pdf

Organización para la Cooperación y el Desarrollo Económicos (OECD) (2013b). La responsabilidad de las personas jurídicas para los delitos de corrupción en América Latina. Recuperado de http://www.oas.org/juridico/PDFs/enc compilacion.pdf

United States v. Kay, 359 F.3d 738 (5th Cir. 2004). 